Amer. Math. Soc. Transl.

(2) Vol. 206, 2002

\title{
Multilinear Spectral Decomposition for Nonlinear Maxwell Equations
}

\author{
A. Babin and A. Figotin \\ This paper is dedicated to M. I. Vishik on the occasion of his 80th birthday.

\begin{abstract}
We study the frequency form, popular in physics, of Maxwell equations for nonlinear spatially inhomogeneous dielectric media. Using wavepackets based on eigenmodes of the underlying linear dielectric medium we develop a mathematically consistent interpretation of the frequency form of nonlinear Maxwell operators. In particular, we construct the operator of nonlinear polarization based on frequency dependent susceptibilities. To this end we use a multilinear variant of the spectral decomposition of selfadjoint operators.
\end{abstract}

\section{Introduction}

This work is motivated by the frequency form (popular in physics; see [1], [2], $[\mathbf{3}],[\mathbf{7}],[\mathbf{8}],[\mathbf{1 0}],[\mathbf{1 1}],[\mathbf{1 2}],[\mathbf{2 2}],[\mathbf{2 3}],[\mathbf{2 6}]$ and references therein) of nonlinear Maxwell equations, including representation of the nonlinear polarization in terms of frequency dependent susceptibilities. Another motivation for this study is our recent work [5] showing the fundamental importance of the spectral theory and frequency composition of waves in understanding of their nonlinear interactions.

Consider first, as an illustration, the case of a homogeneous dielectric medium. In this case, the eigenmodes of the underlying linear medium are just plane waves. The corresponding time harmonic solutions of the linear Maxwell equation are of the form

$$
\mathbf{E}=\mathbf{E}(\mathbf{r}, t)=e^{i(\mathbf{r} \cdot \mathbf{k}-\omega(\mathbf{k}) t)} \mathbf{v},
$$

and a similar expression holds for the magnetic field. Here $\mathbf{r}$ and $t$ are the position vector and the time, respectively, $\mathbf{v}$ is a constant 3-component vector amplitude of the electric field $\mathbf{E}, \mathbf{k}$ is the wavevector, and $\omega(\mathbf{k})$ is the corresponding eigenfrequency.

2000 Mathematics Subject Classification. Primary 78A60, 78M35, 78A40.

Key words and phrases. Maxwell equations, nonlinear susceptibilities, inhomogenious dielectric media, spectral decomposition, multilinear operators. 
Nonlinearity comes to the medium through its constitutive relations, particularly through the polarization $\mathbf{P}$ induced by the electric field $\mathbf{E}$,

$$
\mathbf{P}=\varepsilon^{(\mathbf{1})} \mathbf{E}+\alpha \mathbf{P}_{\mathrm{NL}}(\mathbf{E}),
$$

where $\epsilon^{(\mathbf{1})}$ is the tensor of dielectric permittivity and $\mathbf{E}$ is the electric field. The linear part $\varepsilon^{(\mathbf{1})} \mathbf{E}$ of the polarization determines the properties of the underlying linear medium governed by corresponding linear Maxwell equations, whereas the nonlinear part $\alpha \mathbf{P}_{\mathrm{NL}}(\mathbf{E})$ is responsible for nonlinear effects. The parameter $\alpha$ scales the nonlinear component of the medium to the linear one, and, as in [5], we assume that $\alpha$ is small, which corresponds to the assumption of smallness of the initial excitation currents.

In the classical nonlinear optics (see, for example, $[\mathbf{1 0}],[\mathbf{1 1}],[\mathbf{1 2}]$ ) the nonlinear polarization is of the form of convergent power series

$$
\mathcal{P}_{\mathrm{NL}}(\mathbf{E}(\cdot))=\sum_{h=2}^{\infty} \mathcal{P}^{(h)}(\mathbf{E}(\cdot))
$$

where $\mathcal{P}^{(2)}$ is a bounded quadratic operator, $\mathcal{P}^{(3)}$ is cubic, etc. The $h$-linear operators $\mathcal{P}^{(h)}(\mathbf{E})(\mathbf{r}, t)$ are assumed to satisfy the causality requirement. This leads, in the special case of $h=2$, to the integral operators of the form

$$
\mathcal{P}^{(2)}(\mathbf{E})(\mathbf{r}, t)=\int_{-\infty}^{t} \int_{-\infty}^{t} d t_{1} d t_{2} \mathbf{P}^{(2)}\left(t-t_{1}, t-t_{2}\right)\left(\mathbf{E}\left(\mathbf{r}, t_{1}\right), \mathbf{E}\left(\mathbf{r}, t_{2}\right)\right),
$$

where for every fixed $s_{1}, s_{2} \geq 0$ the term $\mathbf{P}^{(2)}\left(s_{1}, s_{2}\right)$ is a symmetric bilinear mapping from $\mathbb{R}^{3} \times \mathbb{R}^{3}$ into $\mathbb{R}^{3}$, i.e.,

$$
\mathbf{P}^{(2)}\left(s_{1}, s_{2}\right)\left(\mathbf{E}_{1}, \mathbf{E}_{2}\right)=\mathbf{P}^{(2)}\left(s_{2}, s_{1}\right)\left(\mathbf{E}_{2}, \mathbf{E}_{1}\right) .
$$

For $h=2,3, \ldots, h$-linear operators $\mathcal{P}^{(h)}(\cdot)$ are defined by similar formulas

$$
\mathcal{P}^{(h)}\left(\mathbf{E}_{1}, \ldots, \mathbf{E}_{h}\right)=\int_{-\infty}^{t} \ldots \int_{-\infty}^{t} \prod_{j=1}^{h} d t_{j} \mathbf{P}^{(h)}\left(t-t_{1}, \ldots, t-t_{h}\right) \prod_{j=1}^{h} \mathbf{E}_{j}\left(\mathbf{r}, t_{j}\right)
$$

where $\mathbf{P}^{(h)}$ are $h$-linear functions (tensors) from $\left(\mathbb{R}^{3}\right)^{h}$ into $\mathbb{R}^{3}$. The terms $\mathbf{P}^{(h)}$ are assumed to decay (often exponentially) in $t-t_{1}, \ldots, t-t_{h}$, and to be symmetric with respect to permutations $\left\langle t_{i}, \mathbf{E}_{i}\right\rangle \leftrightarrow\left\langle t_{j}, \mathbf{E}_{j}\right\rangle$. We write the vector arguments of $\mathbf{P}^{(h)}$ as $\prod_{j=1}^{h} \mathbf{E}\left(\mathbf{r}, t_{j}\right)$ to indicate that $\mathbf{P}^{(h)}$ is $h$-linear.

Let us look more closely at the quadratic term with $h=2$. In this case $\mathcal{P}^{(2)}(\mathbf{E})=\mathcal{P}^{(2)}(\mathbf{E}, \mathbf{E})$ is a bilinear operator. Consider two plane waves

$$
\mathbf{E}_{j}\left(\mathbf{r}, t_{j}\right)=e^{i\left(\mathbf{r} \cdot \mathbf{k}^{(j)}-\omega\left(\mathbf{k}^{(j)}\right) t_{j}\right)} \mathbf{v}_{j}, \quad j=1,2 .
$$

When two plane waves are superposed, that is, $\mathbf{E}=\mathbf{E}_{1}+\mathbf{E}_{2}$, where $\mathbf{E}_{1}, \mathbf{E}_{2}$ are the plane waves, we get

$$
\mathcal{P}^{(2)}\left(\mathbf{E}_{1}+\mathbf{E}_{2}, \mathbf{E}_{1}+\mathbf{E}_{2}\right)=\mathcal{P}^{(2)}\left(\mathbf{E}_{1}, \mathbf{E}_{1}\right)+2 \mathcal{P}^{(2)}\left(\mathbf{E}_{1}, \mathbf{E}_{2}\right)+\mathcal{P}^{(2)}\left(\mathbf{E}_{2}, \mathbf{E}_{2}\right) .
$$

Here $\mathcal{P}^{(2)}\left(\mathbf{E}_{1}, \mathbf{E}_{1}\right)$ describes the result of the interaction of the wave $\mathbf{E}_{1}$ with itself, $\mathcal{P}^{(2)}\left(\mathbf{E}_{1}, \mathbf{E}_{2}\right)$ describes the interaction of $\mathbf{E}_{1}$ and $\mathbf{E}_{2}$, etc. 
Let us consider now the interaction of a pair of plane waves in more detail. The quadratic polarization is given by

$$
\begin{aligned}
\mathcal{P}^{(2)}\left(\mathbf{E}_{1}, \mathbf{E}_{2}\right)=\int_{-\infty}^{t} \int_{-\infty}^{t} \mathbf{P}^{(2)}\left(t-t_{1}, t-t_{2}\right) \\
\quad \times e^{i\left(\mathbf{r} \cdot\left(\mathbf{k}^{(1)}+\mathbf{k}^{(2)}\right)\right)} e^{-i\left(\omega\left(\mathbf{k}^{(1)}\right) t_{1}+\omega\left(\mathbf{k}^{(2)}\right) t_{2}\right)} \mathbf{v}_{1} \mathbf{v}_{2} d t_{1} d t_{2} .
\end{aligned}
$$

It can be rewritten in the form

$$
\begin{aligned}
& e^{i\left(\mathbf{r} \cdot\left(\mathbf{k}^{(1)}+\mathbf{k}^{(2)}\right)\right)} e^{-i\left(\omega\left(\mathbf{k}^{(1)}\right) t+\omega\left(\mathbf{k}^{(2)}\right) t\right)} \\
& \quad \times \int_{-\infty}^{t} \int_{-\infty}^{t} e^{i\left(\omega\left(\mathbf{k}^{(1)}\right)\left(t-t_{1}\right)+\omega\left(\mathbf{k}^{(2)}\right)\left(t-t_{2}\right)\right)} \mathbf{P}^{(2)}\left(t-t_{1}, t-t_{2}\right) \mathbf{v}_{1} \mathbf{v}_{2} d t_{1} d t_{2} .
\end{aligned}
$$

Clearly, the integral in this formula is the Fourier transform of $\mathbf{P}^{(2)}\left(s_{1}, s_{2}\right)$ extended by zero to negative $s_{1}, s_{2}$. Therefore

$$
\begin{aligned}
\mathcal{P}^{(2)} & \left(\mathbf{E}_{1}, \mathbf{E}_{2}\right) \\
& =e^{-i\left(\omega\left(\mathbf{k}^{(1)}\right)+\omega\left(\mathbf{k}^{(2)}\right)\right) t} e^{i\left(\mathbf{r} \cdot\left(\mathbf{k}^{(1)}+\mathbf{k}^{(2)}\right)\right)} \chi^{(2)}\left(\omega\left(\mathbf{k}^{(1)}\right), \omega\left(\mathbf{k}^{(2)}\right)\right) \mathbf{v}_{1} \mathbf{v}_{2},
\end{aligned}
$$

where $\chi^{(2)}\left(\omega_{1}, \omega_{2}\right)(\cdot)$ is the Fourier transform of $\mathbf{P}_{0}^{(2)}\left(t_{1}, t_{2}\right)(\cdot), \mathbf{P}_{0}^{(2)}\left(t_{1}, t_{2}\right)=$ $\mathbf{P}^{(2)}\left(t_{1}, t_{2}\right)$ for $t_{1}, t_{2} \geq 0$, and $\mathbf{P}_{0}^{(2)}\left(t_{1}, t_{2}\right)=0$ otherwise:

$$
\chi^{(2)}\left(\omega_{1}, \omega_{2}\right)=\int_{-\infty}^{\infty} \int_{-\infty}^{\infty} e^{i\left(\omega_{1} t_{1}+\omega_{2} t_{2}\right)} \mathbf{P}_{0}^{(2)}\left(t_{1}, t_{2}\right) d t_{1} d t_{2} .
$$

The tensor $\chi^{(2)}\left(\omega\left(\mathbf{k}^{(1)}\right), \omega\left(\mathbf{k}^{(2)}\right)\right)$ is called the nonlinear susceptibility. Formula (0.3) can be given a simple interpretation: the waves with wavevectors $\mathbf{k}^{(1)}, \mathbf{k}^{(2)}$ and frequencies $\omega\left(\mathbf{k}^{(1)}\right), \omega\left(\mathbf{k}^{(2)}\right)$ induce the medium polarization wave $\mathcal{P}^{(2)}\left(\mathbf{E}_{1}, \mathbf{E}_{2}\right)$ with the wavevector $\mathbf{k}^{(3)}=\mathbf{k}^{(1)}+\mathbf{k}^{(2)}$, the frequency $\omega=\omega\left(\mathbf{k}^{(1)}\right)+\omega\left(\mathbf{k}^{(2)}\right)$ and the vector amplitude $\chi^{(2)}\left(\omega_{1}, \omega_{2}\right) \mathbf{v}_{1} \mathbf{v}_{2}$. Note that (0.3) includes the action of the tensor $\chi^{(2)}\left(\omega_{1}, \omega_{2}\right)(\cdot)$ on constant vector amplitudes $\mathbf{v}_{1}, \mathbf{v}_{2}$ of the carrier waves resulting in a new vector amplitude $\chi^{(2)}\left(\omega_{1}, \omega_{2}\right) \mathbf{v}_{1} \mathbf{v}_{2}$. It is important that $\chi^{(2)}\left(\omega_{1}, \omega_{2}\right)$ depends on the frequencies $\omega_{1}, \omega_{2}$, whereas $\mathbf{v}_{1}, \mathbf{v}_{2}$ do not depend on time and on the frequencies. Therefore the quadratic operator $\chi^{(2)}\left(\omega_{1}, \omega_{2}\right): \mathbb{R}^{3} \times \mathbb{R}^{3} \rightarrow \mathbb{R}^{3}$ is well defined as acting on the vector amplitudes. This operator is time independent and depends on frequencies as parameters. These properties of the susceptibility are used in nonlinear optics to write differential equations for the dynamics of the vector amplitudes $\mathbf{v}_{1}, \mathbf{v}_{2}$ when they are not constant, but slowly varying in time (the slow-envelope approximation). These equations use $\chi^{(2)}\left(\omega_{1}, \omega_{2}\right)$ with fixed $\omega_{1}$, $\omega_{2}$. Note that in nonlinear optics the notation $\chi^{(2)}\left(\omega_{1}+\omega_{2} ; \omega_{1}, \omega_{2}\right)$ is often used. The reason for this notation is that $\chi^{(2)}$ describes the impact of the waves with the frequencies $\omega_{1}, \omega_{2}$ onto the wave with the frequency $\omega_{1}+\omega_{2}$.

Observe that the above argument gives an interpretation of the nonlinear mechanism of the polarization induced by time and space harmonic waves. Mathematically consistent approach to the same problem requires one to take into account that real waves propagating in a nonlinear medium, being solutions to the nonlinear Maxwell equation, are neither time nor space harmonic. This consistent approach is based on an appropriate asymptotic analysis (see [5], [6]) yielding, in particular, a satisfactory interpretation of the frequency dependent susceptibilities. 
According to $[\mathbf{5}],[\mathbf{6}]$, the medium first nonlinear response is given by

$$
\mathbf{U}^{(1)}(t)=\alpha \int_{-\infty}^{t} e^{\left[-i \mathbf{M}\left(t-t^{\prime}\right)\right]} \mathcal{F}_{\mathrm{NL}}^{\left(h_{0}\right)}\left(\mathbf{U}^{(0)}\right)\left(t^{\prime}\right) d t^{\prime},
$$

where (i) $\mathcal{F}_{\mathrm{NL}}(\mathbf{U})$ is a nonlinear operator whose construction is based on the nonlinear Maxwell equation. This operator has the form similar to the operator $\mathbf{P}_{\mathrm{NL}}(\mathbf{E})$ given by (0.1); (ii) $\mathbf{M}$ is the linear Maxwell operator; (iii) the field $\mathbf{U}^{(0)}(r, t)$ is the medium linear response, i.e., the solution to the linear Maxwell equation, when the excitation current has the form of a wavepacket. We have shown in [5], [6] that for periodic media the principal term of the asymptotic approximation of $\mathbf{U}^{(1)}(r, t)$ can be expressed in terms of the susceptibilities. The relation (0.5) points again to the need for a modification of the elementary derivation of (0.3), based on time and space harmonicity of $\mathbf{E}_{1}, \mathbf{E}_{2}$. Namely, in the general inhomogeneous case $\mathbf{U}^{(0)}$ should be used instead of plane waves and $\mathbf{U}^{(0)}(\mathbf{r}, t)$ is neither time nor space harmonic.

Formally, one can still determine the susceptibility tensor $\chi^{(2)}\left(\mathbf{r} ; \omega_{1}, \omega_{2}\right)$ by formula (0.4) in the general case where $\mathbf{P}_{0}^{(2)}\left(t_{1}, t_{2}\right)=\mathbf{P}_{0}^{(2)}\left(\mathbf{r} ; t_{1}, t_{2}\right)$ depends on the position vector $\mathbf{r}$, and the Maxwell operator has $\mathbf{r}$-dependent coefficients. We have to define how the susceptibility $\chi^{(2)}\left(\omega_{1}, \omega_{2} ; \cdot\right)=\chi^{(2)}\left(\mathbf{r} ; \omega_{1}, \omega_{2} ; \cdot\right)$ determines the action of an operator on the vectors of a Hilbert function space $\mathcal{H}$ related to the linear Maxwell operator $\mathbf{M}$.

In other words, we would like to construct an operator $\hat{\chi}^{(2)}$ starting from the "symbol" $\chi^{(2)}\left(\omega_{1}, \omega_{2}\right)$. This problem is, in a way, similar to constructing a pseudodifferential operator from its symbol. As (0.5) suggests, the action of the operator $\widehat{\chi}^{(2)}$ must depend not only on the symbol $\chi^{(2)}$, but also on the Maxwell operator $\mathbf{M}$. Note also that $\chi^{(2)}\left(\omega_{1}, \omega_{2}\right)$ must act only on field components at the frequencies $\omega_{1}, \omega_{2}$ of $\mathbf{M}$. As is well known from the spectral theory of selfadjoint operators for a general selfadjoint operator $\mathbf{M}$ one has to use the so-called resolution of identity $\mathcal{E}(d \omega)$, which is a projection-valued measure on the frequency axis $\omega$, without much information on generalized eigenfunctions. An additional problem is that we would have to control not only eigenfunctions, but also their pointwise products arising from the nonlinearity. Thus, the main subject of this paper is to develop a mathematically consistent operator approach to the construction of the (quadratic) susceptibility operator corresponding to the (quadratic) symbol $\chi^{(2)}\left(\omega_{1}, \omega_{2}\right)$, which from now on will be called the susceptibility symbol.

In the spatially periodic case one can use the Floquet-Bloch spectral theory to construct the susceptibility operator [5]. To extend the approach of [5] to the case of a general medium we consider the solutions to the underlying linear problem generated by the excitation currents of a special structure. Namely, the excitation currents are assumed to be wavepackets with amplitudes slowly varying in time. Using that approach we will show how one can construct the nonlinear operator susceptibilities $\mathcal{P}_{\chi}^{(h)}$ in terms of the Fourier transforms $\chi^{(h)}$ of the related forms $\mathbf{P}^{(h)}$ and the spectral decomposition of the linear Maxwell operator $\mathbf{M}$ (Theorems $5.2,5.3$ and 5.7).

Let us first consider $\mathbf{P}^{(2)}$ and define $\mathcal{P}_{\chi}^{(2)}\left(\mathbf{v}_{1}, \mathbf{v}_{2}\right)$ as

$$
\mathcal{P}_{\chi}^{(2)}\left(\mathbf{v}_{1}, \mathbf{v}_{2}\right)(t)=\int_{-\infty}^{\infty} \int_{-\infty}^{\infty} e^{-i\left(\omega_{1}+\omega_{2}\right) t} \chi^{(2)}\left(\omega_{1}, \omega_{2} ; d \mathcal{E}\left(\omega_{1}\right) \mathbf{v}_{1}, d \mathcal{E}\left(\omega_{2}\right) \mathbf{v}_{2}\right)
$$


where $\chi^{(2)}\left(\omega_{1}, \omega_{2} ; \cdot\right)$ is the Fourier transform of $\mathbf{P}_{0}^{(2)}\left(t_{1}, t_{2}\right)$. This form is a direct generalization of (0.3); an element of a corresponding integral sum is of the form

$$
e^{-i\left(\omega_{1}+\omega_{2}\right) t} \chi^{(2)}\left(\omega_{1}, \omega_{2} ;\left[\mathcal{E}\left(\omega_{1}^{\prime}\right)-\mathcal{E}\left(\omega_{1}^{\prime \prime}\right)\right] \mathbf{v}_{1},\left[\mathcal{E}\left(\omega_{2}^{\prime}\right)-\mathcal{E}\left(\omega_{2}^{\prime \prime}\right)\right] \mathbf{v}_{2}\right),
$$

with $\omega_{1} \in\left[\omega_{1}^{\prime}, \omega_{1}^{\prime \prime}\right], \omega_{2} \in\left[\omega_{2}^{\prime}, \omega_{2}^{\prime \prime}\right]$. The bilinear operator $\chi^{(2)}\left(\omega_{1}, \omega_{2} ; \cdot\right)$ depends on the frequencies $\omega_{1}$ and $\omega_{2}$ and acts on the vectors $\left[\mathcal{E}\left(\omega_{1}^{\prime}\right)-\mathcal{E}\left(\omega_{1}^{\prime \prime}\right)\right] \mathbf{v}_{1}$ and $\left[\mathcal{E}\left(\omega_{2}^{\prime}\right)-\mathcal{E}\left(\omega_{2}^{\prime \prime}\right)\right] \mathbf{v}_{2}$; the susceptibility operator $\chi^{(2)}\left(\omega_{1}, \omega_{2} ; \cdot\right)$ is time independent. Note that in (0.3) the action of $\chi^{(2)}$ on the vector amplitudes $\mathbf{v}_{1}, \mathbf{v}_{2}$ of the waves ignores the $r$-dependency of the waves, because the $r$-dependency occurs only in scalar coefficients of the vectors. In contrast with that, vectors $\left[\mathcal{E}\left(\omega_{1}^{\prime}\right)-\mathcal{E}\left(\omega_{1}^{\prime \prime}\right)\right] \mathbf{v}_{1}$ in (0.6) are rather general elements of a Hilbert function space of $r$-dependent vector-valued functions, and one cannot separate the spatial structure from the vector structure of eigenfunctions in general media. Therefore, from now on we will refer to $\chi^{(2)}\left(\omega_{1}, \omega_{2} ; \cdot\right)$ as operator susceptibilities. In the case of a spatially periodic medium one can separate large-scale spatial dependence using the Floquet-Bloch theory (for details see [5] and the last section of this article).

For the general case of $h>2$ and a selfadjoint $\mathbf{M}$ the symbol $\chi^{(h)}\left(\omega_{1}, \ldots, \omega_{h}\right.$; $\left.\mathbf{v}_{1}, \ldots, \mathbf{v}_{h}\right)$ is a multilinear operator which depends on real parameters $\omega_{1}, \ldots, \omega_{h}$ (frequencies). In this case we define in a similar fashion in Section 5 the operator $\mathcal{A}_{\mathbf{M}, \chi}$ based on $\chi^{(h)}$ and $\mathbf{M}$.

In summary, one of the main objectives of this paper is to give a precise meaning to the integrals (0.6) which also can be looked at as multilinear spectral decompositions. This is done in Theorem 5.3; Theorem 5.7 describes the relation of such multilinear spectral decompositions with the response of the media given by integrals of the form (0.5).

\section{Multilinear operators in Sobolev spaces}

In this section we consider $n$-component vector-valued functions (vector fields) that depend on $d$ spatial variables in the entire $\mathbb{R}^{d}$. For the classical Maxwell equations $n=3, d=1,2,3$. We focus primarily on the case $d=3$.

In our studies we use the following functional spaces:

$\mathbf{W}_{2}^{s}=\mathbf{W}_{2}^{s}\left(\mathbb{R}^{d}\right), s=1,2, \ldots$, is the Sobolev space of vector fields $\mathbf{V}(\mathbf{r}), \mathbf{r} \in \mathbb{R}^{d}$, with the norm

$$
\begin{aligned}
\|\mathbf{V}\|_{\mathbf{W}_{2}^{s}\left(\mathbb{R}^{d}\right)}^{2} & =\sum_{0 \leq l_{1}+\cdots+l_{d} \leq s} \int_{\mathbb{R}^{d}}\left|\partial_{1}^{l_{1}} \cdots \partial_{d}^{l_{d}} \mathbf{V}(\mathbf{r})\right|^{2} \mathbf{d r}, \\
|\mathbf{V}| & =\left(\left|V_{1}\right|^{2}+\cdots+\left|V_{n}\right|^{2}\right)^{1 / 2} .
\end{aligned}
$$

$\mathcal{C}^{s}\left(\mathbb{R}^{d}\right)$ is the space of $s$ times continuously differentiable and uniformly bounded vector fields with the norm

$$
\|\mathbf{V}\|_{\mathcal{C}^{s}\left(\mathbb{R}^{d}\right)}^{2}=\sup _{l_{1}+\cdots+l_{d} \leq s, \mathbf{r} \in \mathbb{R}^{d}}\left|\partial_{1}^{l_{1}} \cdots \partial_{d}^{l_{d}} \mathbf{V}(\mathbf{r})\right|, s=1,2, \ldots
$$

$\mathbf{W B}_{2}^{s}\left(\mathbb{R}^{d}\right)$ is the space of functions that locally belong to $\mathbf{W}_{2}^{s}\left(\mathbb{R}^{d}\right)$ and their local $\mathbf{W}_{2}^{s}$-norms are uniformly bounded,

$$
\|\mathbf{V}\|_{\mathbf{W B}_{2}^{s}\left(\mathbb{R}^{d}\right)}^{2}=\sup _{\mathbf{y} \in \mathbb{R}^{d}} \sum_{0 \leq l_{1}+\cdots+l_{d} \leq s} \int_{|r| \leq 1}\left|\partial_{1}^{l_{1}} \cdots \partial_{d}^{l_{d}} \mathbf{V}(\mathbf{r}+\mathbf{y})\right|^{2} \mathbf{d r}, s=1,2, \ldots
$$


The nonlinear Maxwell equations considered in the next section involve nonlinear operators in the form of a series,

$$
\mathcal{S}_{\mathrm{NL}}(\mathbf{r}, t ; \mathbf{D})=\mathcal{S}^{\left(h_{0}\right)}(\mathbf{r}, t ; \mathbf{D})+\alpha \sum_{h>h_{0} \geq 2} \mathcal{S}^{(h)}(\mathbf{r}, t ; \mathbf{D}),
$$

convergence of which is discussed below. The term $\mathcal{S}^{(h)}(\mathbf{r}, t ; \mathbf{D})$ in (1.4) is a multilinear integral operator with the causal time dispersion, namely

$$
\mathcal{S}^{(h)}(\mathbf{r}, t ; \mathbf{D})=\int_{-\infty}^{t} \cdots \int_{-\infty}^{t} \mathbf{S}^{(h)}\left(\mathbf{r} ; t-t_{1}, \ldots, t-t_{h}\right) \prod_{j=1}^{h} \mathbf{D}\left(\mathbf{r}, t_{j}\right) \prod_{j=1}^{h} d t_{j} .
$$

The kernels $\mathbf{S}^{(h)}\left(\mathbf{r} ; t_{1}, \ldots, t_{h}\right)$ are $h$-linear functions (tensors) from $\mathbb{R}^{n h}$ into $\mathbb{R}^{n}$ depending on $\mathbf{r} \in \mathbb{R}^{d}, t_{1}, \ldots, t_{h} \geq 0$. We remind that the action $v=f\left(g_{1}, \ldots, g_{h}\right)$ of a tensor $f$ on variables $g_{1}, \ldots, g_{h} \in \mathbb{R}^{n}$ in the standard basis is represented by the formula

$$
v_{j}=\sum_{i_{1}, \ldots, i_{h}} f_{i_{1}, \ldots, i_{h}}^{j} g_{1 i_{1}} \cdots g_{h i_{h}}
$$

where $f_{i_{1}, \ldots, i_{h}}^{j}$ are the coefficients of $f$ in the standard basis of $\mathbb{R}^{n}$. Hence, the corresponding coefficients of the tensor $\mathbf{S}^{(h)}$ in (1.5) may depend on $\mathbf{r}$.

We denote by $\mathbf{S}_{0}^{(h)}\left(\mathbf{r} ; t_{1}, \ldots, t_{h}\right)$ an extension of $\mathbf{S}^{(h)}\left(\mathbf{r} ; t_{1}, \ldots, t_{h}\right)$ from the set $t_{1}, \ldots, t_{h} \geq 0$ to the entire $\mathbb{R}^{h}$ by zero outside $t_{1}, \ldots, t_{h} \geq 0$.

We assume that for some $s>d / 2$ there exist positive constants $C_{0 S}$ and $C_{1 S}$ such that for all $h$

$$
\int_{0}^{\infty} \cdots \int_{0}^{\infty}\left\|\mathbf{S}^{(h)}\left(\mathbf{r} ; t_{1}, \ldots, t_{h}\right)\right\|_{\mathbf{W B}_{2}^{s}\left(\mathbb{R}^{d}\right)} d t_{1} \cdots d t_{h} \leq C_{0 S} C_{1 S}^{h}
$$

In (1.7) the norm $\left\|\mathbf{S}^{(h)}\left(\mathbf{r} ; t_{1}, \ldots, t_{h}\right)\right\|_{\mathbf{W B}_{2}^{s}\left(\mathbb{R}^{d}\right)}$ of an $h$-linear $\mathbf{r}$-dependent tensor is given by (1.3) in which $|\cdot|$ is understood as the norm of an $h$-linear constant tensor in $\mathbb{R}^{n}$. Namely, when the action $v=f\left(g_{1}, \ldots, g_{h}\right)$ of a tensor $f$ on variables $g_{1}, \ldots, g_{h} \in \mathbb{R}^{n}$ is given by (1.6), we set

$$
|f|=\left[\sum_{j, i_{1}, \ldots, i_{h}}\left|f_{i_{1}, \ldots, i_{h}}^{j}\right|^{2}\right]^{1 / 2}
$$

Recall now that if $\mathcal{B}_{1}, \mathcal{B}_{2}$ are Banach spaces, the operator norm of an $h$-linear operator $\mathcal{P}^{(h)}$ from $\mathcal{B}_{1}^{h}$ into $\mathcal{B}_{2}$ is defined by

$$
\left\|\mathcal{P}^{(h)}\right\|_{\mathcal{B}_{1}, \mathcal{B}_{2}}=\sup _{\left\|\mathbf{u}_{1}\right\|_{\mathcal{B}_{1}} \leq 1, \ldots,\left\|\mathbf{u}_{h}\right\|_{\mathcal{B}_{1}} \leq 1}\left\|\mathcal{P}^{(h)}\left(\mathbf{u}_{1}, \ldots, \mathbf{u}_{h}\right)\right\|_{\mathcal{B}_{2}} .
$$

A multilinear symmetric operator $\mathcal{P}^{(h)}\left(\mathbf{u}_{1}, \ldots, \mathbf{u}_{h}\right)$ from $\mathcal{B}_{1}^{h}$ into $\mathcal{B}_{2}$ generates a polynomial operator $\mathcal{P}^{(h)}(\mathbf{u})$ from $\mathcal{B}_{1}$ into $\mathcal{B}_{2}, \mathcal{P}^{(h)}(\mathbf{u})=\mathcal{P}^{(h)}(\mathbf{u}, \ldots, \mathbf{u})$ (see monograph [17], Sections 3.16-3.19 and the entire Chapter XXVI, and the references therein).

We consider multilinear operators $\mathcal{S}^{(h)}$ defined by (1.5) in the space $\mathcal{B}$ of bounded continuous functions of the time variable with values in the Sobolev space $\mathbf{W}_{2}^{s}\left(\mathbb{R}^{d}\right)$,

$$
\mathcal{B}=\mathcal{C}\left((-\infty, T] ; \mathbf{W}_{2}^{s}\left(\mathbb{R}^{d}\right)\right)
$$


with the norm

$$
\|\mathbf{V}\|_{\mathcal{C}\left((-\infty, T] ; \mathbf{W}_{2}^{s}\left(\mathbb{R}^{d}\right)\right)}=\sup _{t \leq T}\|\mathbf{V}\|_{\mathbf{W}_{2}^{s}\left(\mathbb{R}^{d}\right)}
$$

We will prove that under condition $(1.7) \mathcal{S}^{(h)}$ is a bounded operator in this space and $\mathcal{S}_{\mathrm{NL}}(\mathbf{r}, t ; \mathbf{D})$ is well defined by $(1.4)$ for small $\mathbf{D}$. To do that, we need two lemmas.

First, we prove a lemma on the pointwise multiplication of scalar functions in $\mathbf{W}_{2}^{s}\left(\mathbb{R}^{d}\right)$.

Lemma 1.1. Let $s>d / 2, f \in \mathbf{W}_{2}^{s}\left(\mathbb{R}^{d}\right), g \in \mathbf{W B}_{2}^{s}\left(\mathbb{R}^{d}\right)$. Then

$$
\|f g\|_{\mathbf{W}_{2}^{s}\left(\mathbb{R}^{d}\right)} \leq C_{1}\|f\|_{\mathbf{W}_{2}^{s}\left(\mathbb{R}^{d}\right)}\|g\|_{\mathbf{W B}_{2}^{s}\left(\mathbb{R}^{d}\right)}
$$

where $C_{1}$ depends only on $s$ and $d$.

Proof. We use two well-known inequalities:

$$
\begin{aligned}
\|f g\|_{\mathbf{W}_{2}^{s}\left(\mathbb{R}^{d}\right)} & \leq C_{0}\|f\|_{\mathbf{W}_{2}^{s}\left(\mathbb{R}^{d}\right)}\|g\|_{\mathbf{W}_{2}^{s}\left(\mathbb{R}^{d}\right)}, \\
f & \in \mathbf{W}_{2}^{s}\left(\mathbb{R}^{d}\right), \quad g \in \mathbf{W}_{2}^{s}\left(\mathbb{R}^{d}\right), \\
\|f g\|_{\mathbf{W}_{2}^{s}\left(\mathbb{R}^{d}\right)} & \leq C_{0}\|f\|_{\mathbf{W}_{2}^{s}\left(\mathbb{R}^{d}\right)}\|g\|_{\mathcal{C}^{s}\left(\mathbb{R}^{d}\right)}, \\
f & \in \mathbf{W}_{2}^{s}\left(\mathbb{R}^{d}\right), \quad g \in \mathcal{C}^{s}\left(\mathbb{R}^{d}\right) .
\end{aligned}
$$

If $g \in \mathbf{W B}_{2}^{s}\left(\mathbb{R}^{d}\right)$ and $\phi$ is an infinitely differentiable function with compact support, then $\phi g \in \mathbf{W}_{2}^{s}\left(\mathbb{R}^{d}\right)$. Let $\phi_{0}(\mathbf{r})$ be an infinitely smooth nonnegative function such that

$$
\phi_{0}(\mathbf{r}) \geq 0, \quad \phi_{0}(\mathbf{r})=1 \text { for }|\mathbf{r}| \leq d, \quad \phi(\mathbf{r})=0 \text { for }|\mathbf{r}| \geq 2 d .
$$

We note that the supports of all functions $\phi_{0}(\mathbf{r}-\mathbf{l})$, as 1 runs over the set $\mathbb{Z}^{d}$ of $d$-dimensonal integer-valued vectors $\mathbb{Z}^{d}$, cover the entire $\mathbb{R}^{d}$. In addition,

$$
\Phi(\mathbf{r})=\sum_{\mathbf{l} \in \mathbb{Z}^{d}} \phi_{0}(\mathbf{r}-\mathbf{l})
$$

is an infinitely smooth periodic function and

$$
1 \leq \Phi(\mathbf{r}) \leq(4 d-1)^{d},(4 d-1)^{-d} \leq 1 / \Phi(\mathbf{r}) \leq 1
$$

We also use the function

$$
\phi_{01}(\mathbf{r})=\phi_{0}((\mathbf{r}-\mathbf{l}) /(2 d)) .
$$

Clearly $\phi_{0}((\mathbf{r}-\mathbf{l}) /(2 d)) \phi_{0}(\mathbf{r}-\mathbf{l})=\phi_{0}(\mathbf{r}-\mathbf{l})$ for every $\mathbf{r}$ and $\mathbf{l}$. From (1.11) we get

$$
\begin{aligned}
\|f g\|_{\mathbf{W}_{2}^{s}\left(\mathbb{R}^{d}\right)} & =\|f g \Phi / \Phi\|_{\mathbf{W}_{2}^{s}\left(\mathbb{R}^{d}\right)} \leq C_{0}\|f g \Phi\|_{\mathbf{W}_{2}^{s}\left(\mathbb{R}^{d}\right)}\|1 / \Phi\|_{\mathcal{C}^{s}\left(\mathbb{R}^{d}\right)} \\
& \leq\|1 / \Phi\|_{\mathcal{C}^{s}\left(\mathbb{R}^{d}\right)} C_{0} \sum_{\mathbf{l} \in \mathbb{Z}^{d}}\left\|f g \phi_{0}(\cdot-\mathbf{l})\right\|_{\mathbf{W}_{2}^{s}\left(\mathbb{R}^{d}\right)} \\
& =\|1 / \Phi\|_{\mathcal{C}^{s}\left(\mathbb{R}^{d}\right)} C_{0} \sum_{\mathbf{l} \in \mathbb{Z}^{d}}\left\|f \phi_{01} g \phi_{0}(\cdot-\mathbf{l})\right\|_{\mathbf{W}_{2}^{s}\left(\mathbb{R}^{d}\right)} \\
& \leq\|1 / \Phi\|_{\mathcal{C}^{s}\left(\mathbb{R}^{d}\right)} C_{0}^{2} \sum_{\mathbf{l} \in \mathbb{Z}^{d}}\left\|f \phi_{0 \mathbf{l}}\right\|_{\mathbf{W}_{2}^{s}\left(\mathbb{R}^{d}\right)}\left\|g \phi_{0}(\cdot-\mathbf{l}) \Phi\right\|_{\mathbf{W}_{2}^{s}\left(\mathbb{R}^{d}\right)} .
\end{aligned}
$$

From definitions (1.3) and (1.1) we get

$$
\left\|g \phi_{0}(\cdot-\mathbf{l}) \Phi\right\|_{\mathbf{W}_{2}^{s}\left(\mathbb{R}^{d}\right)} \leq C\|g\|_{\mathbf{W B}_{2}^{s}\left(\mathbb{R}^{d}\right)}
$$


and

$$
\sum_{\mathbf{1} \in \mathbb{Z}^{d}}\left\|f \phi_{01}\right\|_{\mathbf{W}_{2}^{s}\left(\mathbb{R}^{d}\right)} \leq C\|f\|_{\mathbf{W}_{2}^{s}\left(\mathbb{R}^{d}\right)} .
$$

Therefore (1.12) implies (1.9).

Lemma 1.2. Let $f$ be an h-linear tensor in $\mathbb{R}^{n}$ with coefficients that depend on the variable $\mathbf{r} \in \mathbb{R}^{d}$. Assume that the coefficients belong to $\mathbf{W B}_{2}^{s}\left(\mathbb{R}^{d}\right)$ with $s>d / 2$. Then for $g \in\left(\mathbf{W}_{2}^{s}\left(\mathbb{R}^{d}\right)\right)^{h}$

$$
\left\|f\left(g_{1}, \ldots, g_{h}\right)\right\|_{\mathbf{W}_{2}^{s}\left(\mathbb{R}^{d}\right)} \leq C_{2} C_{3}^{h-1}\|f\|_{\mathbf{W B}_{2}^{s}\left(\mathbb{R}^{d}\right)}\left\|g_{1}\right\|_{\mathbf{W}_{2}^{s}\left(\mathbb{R}^{d}\right)} \cdots\left\|g_{h}\right\|_{\mathbf{W}_{2}^{s}\left(\mathbb{R}^{d}\right)},
$$

where $C_{2}, C_{3}$ depend only on $s, n$ and $d$.

Proof. Applying (1.9) and (1.10) to (1.6) we get

$$
\begin{aligned}
\left\|v^{j}\right\|_{\mathbf{W}_{2}^{s}\left(\mathbb{R}^{d}\right)} & \leq \sum_{i_{1}, \ldots, i_{h}} C_{1}\left\|f_{i_{1}, \ldots, i_{h}}^{j}\right\|_{\mathbf{W}_{2}^{s}\left(\mathbb{R}^{d}\right)}\left\|g_{1 i_{1}} \cdots g_{h i_{h}}\right\|_{\mathbf{W}_{2}^{s}\left(\mathbb{R}^{d}\right)} \\
& \leq C_{1} C_{0}^{h-1} \sum_{i_{1}, \ldots, i_{h}}\left\|f_{i_{1}, \ldots, i_{h}}^{j}\right\|_{\mathbf{W}_{2}^{s}\left(\mathbb{R}^{d}\right)}\left\|g_{1 i_{1}}\right\|_{\mathbf{W}_{2}^{s}\left(\mathbb{R}^{d}\right)} \cdots\left\|g_{h i_{h}}\right\|_{\mathbf{W}_{2}^{s}\left(\mathbb{R}^{d}\right)} .
\end{aligned}
$$

Therefore,

$$
\begin{aligned}
& \sum_{j}\left\|v^{j}\right\|_{\mathbf{W}_{2}^{s}\left(\mathbb{R}^{d}\right)}^{2} \\
& \quad \leq\left(C_{1} C_{0}^{h-1}\right)^{2} \sum_{j, i_{1}, \ldots, i_{h}}\left\|f_{i_{1}, \ldots, i_{h}}^{j}\right\|_{\mathbf{W}_{2}^{s}\left(\mathbb{R}^{d}\right)}^{2} \sum_{i_{1}}\left\|g_{1 i_{1}}\right\|_{\mathbf{W}_{2}^{s}\left(\mathbb{R}^{d}\right)}^{2} \cdots \sum_{i_{h}}\left\|g_{h i_{h}}\right\|_{\mathbf{W}_{2}^{s}\left(\mathbb{R}^{d}\right)}^{2} .
\end{aligned}
$$

This implies (1.13).

Recall that an operator $\mathcal{S}$ in a Banach space $\mathcal{B}$ is analytic (at zero) if it can be represented by a convergent series,

$$
\begin{gathered}
\mathcal{S}(\mathbf{u})=\sum_{h=h_{0}}^{\infty} \mathcal{S}^{(h)}(\mathbf{u}), h_{0} \geq 1, \\
\left\|\mathcal{S}^{(h)}\right\|_{\mathcal{B}, \mathcal{B}} \leq c_{0} c_{1}^{h},
\end{gathered}
$$

where every $\mathcal{S}^{(h)}(\mathbf{u})=\mathcal{S}^{(h)}(\mathbf{u}, \ldots, \mathbf{u})$ is an $h$-linear operator, i.e., $\mathcal{S}^{(h)}\left(\mathbf{u}_{1}, \ldots, \mathbf{u}_{h}\right)$ is a linear operator with respect to every $\mathbf{u}_{i}, i=1, \ldots, h$.

Theorem 1.3. Let (1.7) hold with $s>d / 2$. Then $\mathcal{S}^{(h)}$ defined by (1.5) is a bounded $h$-linear operator in $\mathcal{B}=\mathcal{C}\left((-\infty, T] ; \mathbf{W}_{2}^{s}\left(\mathbb{R}^{d}\right)\right)$, and the norm of the operator $\mathcal{S}^{(h)}$ in this space is bounded,

$$
\left\|\mathcal{S}^{(h)}\right\|_{\mathcal{B}, \mathcal{B}} \leq C_{2}^{\prime} C_{3}^{h} \int_{0}^{\infty} \cdots \int_{0}^{\infty}\left\|\mathbf{S}^{(h)}\left(\mathbf{r} ; t_{1}, \ldots, t_{h}\right)\right\|_{\mathbf{W B}_{2}^{s}\left(\mathbb{R}^{d}\right)} d t_{1} \cdots d t_{h},
$$

where $C_{2}^{\prime}, C_{3}$ do not depend on $T$. The series (1.4) determines an analytic operator $\mathcal{S}_{N L}$ in the neighborhood of zero in the space $\mathcal{C}\left((-\infty, T] ; \mathbf{W}_{2}^{s}\left(\mathbb{R}^{d}\right)\right)$, and the radius of convergence of this operator does not depend on $T$. 
Proof. We consider a multilinear operator that corresponds to (1.5):

$$
\mathcal{S}^{(h)}\left(t ; \mathbf{D}_{1}, \ldots, \mathbf{D}_{h}\right)=\int_{-\infty}^{t} \ldots \int_{-\infty}^{t} \mathbf{S}^{(h)}\left(t-t_{1}, \ldots, t-t_{h}\right) \prod_{j=1}^{h} \mathbf{D}_{j}\left(t_{j}\right) \prod_{j=1}^{h} d t_{j} .
$$

We have

$$
\begin{aligned}
& \left\|\mathcal{S}^{(h)}\left(t ; \mathbf{D}_{1}, \ldots, \mathbf{D}_{1}\right)\right\|_{\mathbf{W}_{2}^{s}\left(\mathbb{R}^{d}\right)} \\
& \quad \leq \int_{-\infty}^{t} \ldots \int_{-\infty}^{t}\left\|\mathbf{S}^{(h)}\left(t-t_{1}, \ldots, t-t_{h}\right) \prod_{j=1}^{h} \mathbf{D}_{j}\left(t_{j}\right)\right\|_{\mathbf{W}_{2}^{s}\left(\mathbb{R}^{d}\right)} \prod_{j=1}^{h} d t_{j} .
\end{aligned}
$$

By (1.13)

$$
\begin{aligned}
& \left\|\mathbf{S}^{(h)}\left(t-t_{1}, \ldots, t-t_{h}\right) \prod_{j=1}^{h} \mathbf{D}_{j}\left(t_{j}\right)\right\|_{\mathbf{W}_{2}^{s}\left(\mathbb{R}^{d}\right)} \\
& \quad \leq C_{2} C_{3}^{h-1}\left\|\mathbf{S}^{(h)}\left(t-t_{1}, \ldots, t-t_{h}\right)\right\|_{\mathbf{W B}_{2}^{s}\left(\mathbb{R}^{d}\right)} \prod_{j=1}^{h}\left\|\mathbf{D}_{j}\left(t_{j}\right)\right\|_{\mathbf{W}_{2}^{s}\left(\mathbb{R}^{d}\right)} .
\end{aligned}
$$

Therefore, when $t \leq T$,

$$
\begin{aligned}
& \left\|\mathcal{S}^{(h)}\left(t ; \mathbf{D}_{1}, \ldots, \mathbf{D}_{1}\right)\right\|_{\mathbf{W}_{2}^{s}\left(\mathbb{R}^{d}\right)} \\
& \leq C_{2} C_{3}^{h-1} \int_{-\infty}^{t} \cdots \int_{-\infty}^{t}\left\|\mathbf{S}^{(h)}\left(t-t_{1}, \ldots, t-t_{h}\right)\right\|_{\mathbf{W B}_{2}^{s}\left(\mathbb{R}^{d}\right)} \prod_{j=1}^{h} d t_{j} \\
& \quad \times \prod_{j=1}^{h} \sup _{t_{j} \leq T}\left\|\mathbf{D}_{j}\left(t_{j}\right)\right\|_{\mathbf{W}_{2}^{s}\left(\mathbb{R}^{d}\right)} .
\end{aligned}
$$

This implies (1.16). From (1.7) and (1.16) it follows that the series (1.4) converges.

\section{Motivation of the problem}

In this section we discuss some results of [5] and [6] to motivate our choices of operators and problems. The statements of this section are not used in the proofs of the following sections.

Following [5], we write the linear Maxwell equations in the operator form

$$
\partial_{t} \mathbf{U}=-i \mathbf{M U}-\mathbf{J},
$$

where

$$
\mathbf{U}=\left[\begin{array}{l}
\mathbf{D} \\
\mathbf{B}
\end{array}\right], \mathbf{M U}=i\left[\begin{array}{c}
\nabla \times \mathbf{B} \\
-\nabla \times\left(\eta^{(\mathbf{1})}(\mathbf{r}) \mathbf{D}\right)
\end{array}\right], \mathbf{J}=4 \pi\left[\begin{array}{c}
\mathbf{J}_{E} \\
\mathbf{J}_{M}
\end{array}\right] .
$$

Here $\eta^{(1)}(\mathbf{r})$ is the impermeability tensor, and the spatial variable is $\mathbf{r} \in \mathbb{R}^{d}$, where we consider $d=1,2,3$. The matrix $\eta^{(1)}$ is a positive definite symmetric $3 \times 3$ matrix with real entries. We assume that it satisfies the following condition:

$$
C_{-} \mathbf{1} \leq \eta^{(1)}(\mathbf{r}) \leq C_{+} \mathbf{1} \text {, where } 0<C_{-} \leq C_{+} .
$$


Everywhere in this section we assume that the elements of the matrix $\eta^{(1)}(\mathbf{r})$ are bounded in $\mathbf{W B}_{2}^{s}\left(\mathbb{R}^{d}\right), s>d / 2$. The equations (2.1) are assumed to be written in a dimensionless form. In particular, the light speed is equal to one. We use electric and magnetic inductances $\mathbf{D}$ and $\mathbf{B}$ as basic field variables rather than the electric and magnetic fields $\mathbf{E}$ and $\mathbf{H}$. We assume that all the 3-component vector fields $\mathbf{D}, \mathbf{B}, \mathbf{J}_{E}$ and $\mathbf{J}_{M}$ are divergence free,

$$
\nabla \cdot \mathbf{D}=\nabla \cdot \mathbf{B}=\nabla \cdot \mathbf{J}_{E}=\nabla \cdot \mathbf{J}_{M}=0
$$

Let us consider now some important properties of the linear Maxwell equations (2.1). The linear operator $\mathbf{M}$ defined by (2.2) is selfadjoint in the Hilbert space $\mathcal{H}$ consisting of 6 -component vector fields with the scalar product

$$
(\mathbf{u}, \mathbf{v})_{\mathcal{H}}=\int_{\mathbb{R}^{d}}(\mathbf{u}(\mathbf{r}), \mathbf{v}(\mathbf{r}))_{\sigma} d \mathbf{r},
$$

where

$$
(\mathbf{u}(\mathbf{r}), \mathbf{v}(\mathbf{r}))_{\sigma}=\mathbf{u}^{*}(\mathbf{r}) \cdot \sigma(\mathbf{r}) \mathbf{v}(\mathbf{r}), \quad \sigma(\mathbf{r})=\left[\begin{array}{cc}
\eta^{(1)}(\mathbf{r}) & \mathbf{0} \\
\mathbf{0} & \mathbf{I}
\end{array}\right]
$$

(see [9], [15] for details). In view of the condition (2.3), the norm $\|\cdot\|_{\mathcal{H}}$ is equivalent to the standard Lebesgue $L_{2}$-norm.

Let us also introduce a sequence of Hilbert spaces $\mathcal{H}^{s}, s=0,1, \ldots$, consisting of 6-component vector fields $\mathbf{V}(\mathbf{r})=\left[\begin{array}{l}\mathbf{D}(\mathbf{r}) \\ \mathbf{B}(\mathbf{r})\end{array}\right]$ satisfying (2.4) with the norm defined by

$$
\|\mathbf{V}\|_{\mathcal{H}^{s}}=\left(\int_{\mathbb{R}^{d}}\left|\mathbf{M}^{s} \mathbf{V}(\mathbf{r})\right|_{\sigma}^{2} d \mathbf{r}+\int_{\mathbb{R}^{d}}|\mathbf{V}(\mathbf{r})|_{\sigma}^{2} d \mathbf{r}\right)^{1 / 2}, s=0,1, \ldots
$$

Lemma 2.1. If (2.3) holds and all elements of the matrix $\eta^{(1)}(\mathbf{r})$ are bounded in $\mathbf{W B}_{2}^{s}\left(\mathbb{R}^{d}\right), s>d / 2$, then the Maxwell norm $\|\mathbf{V}\|_{\mathcal{H}^{s}}$ and Sobolev norm $\|\mathbf{V}\|_{\mathbf{W}_{2}^{s}}$ are equivalent on the space $\mathcal{H}^{s}$ consisting of 6-component vector fields $\mathbf{V}(\mathbf{r})$ that satisfy (2.4):

$$
c\|\mathbf{V}\|_{\mathbf{W}_{2}^{s}} \leq\|\mathbf{V}\|_{\mathcal{H}^{s}} \leq C\|\mathbf{V}\|_{\mathbf{W}_{2}^{s}} .
$$

The proof of this lemma uses the ellipticity of $\mathbf{M}$ on divergence-free fields, and is to appear in $[6]$.

From Lemma 2.1 and Theorem 1.3 we obtain the following theorem.

TheOREM 2.2. Let (1.7) hold with $s>d / 2$. Then $\mathcal{S}^{(h)}$ is a bounded h-linear operator in $\mathcal{B}^{s}=\mathcal{C}\left((-\infty, T] ; \mathcal{H}^{s}\right)$; the norm of the operator $\mathcal{S}^{(h)}$ in this space is bounded,

$$
\left\|\mathcal{S}^{(h)}\right\|_{\mathcal{B}^{s}, \mathcal{B}^{s}} \leq C_{4} C_{5}^{h} \int_{-\infty}^{0} \cdots \int_{-\infty}^{0}\left\|\mathbf{S}^{(h)}\left(\mathbf{r} ; t_{1}, \ldots, t_{h}\right)\right\|_{\mathbf{W B}_{2}^{s}\left(\mathbb{R}^{d}\right)} d t_{1} \cdots d t_{h},
$$

where $C_{4}, C_{5}$ do not depend on $T$. The series (1.4) determines an analytic operator $\mathcal{S}_{N L}$ in the neighborhood of zero in the space $\mathcal{C}\left((-\infty, T] ; \mathcal{H}^{s}\right)$, and the radius of convergence of this operator does not depend on $T$.

Since the operator $\mathbf{M}$ is selfadjoint in $\mathcal{H}^{s}$ for every $s$, the operators $e^{[-i \mathbf{M} t]}$, $t \in \mathbb{R}$, form a one-parameter group of unitary operators in $\mathcal{H}^{s}$. The exponent can be written explicitly in terms of the spectral projections. 
Following [5], we recast the nonlinear Maxwell equations in the following concise form:

$$
\partial_{t} \mathbf{U}=-i \mathbf{M} \mathbf{U}+\alpha \mathcal{F}_{\mathrm{NL}}(\mathbf{U})-\mathbf{J} ; \mathbf{U}(t)=0 \text { for } t \leq 0,
$$

where

$$
\mathcal{F}_{\mathrm{NL}}(\mathbf{U})=\left[\begin{array}{c}
\mathbf{0} \\
\nabla \times \mathcal{S}_{\mathrm{NL}}(\mathbf{r}, t ; \mathbf{D})
\end{array}\right], \mathbf{U}=\left[\begin{array}{c}
\mathbf{D} \\
\mathbf{B}
\end{array}\right]
$$

where $\mathcal{S}_{\mathrm{NL}}(\mathbf{r}, t ; \mathbf{D}(\mathbf{r}, t))$ is defined by $(1.4)$.

We assume that the medium is at rest for all negative times by requiring the impressed currents $\mathbf{J}$ to vanish for all negative times, i.e.,

$$
\mathbf{J}(t)=0 \text { for } t \leq 0
$$

Observe that (2.9) shows that the parameter $\alpha$ determines the relative magnitude of the nonlinearity, and, in particular, if $\alpha=0$, the equation becomes linear.

It is convenient to rewrite $(2.9)$ in the integral form

$$
\mathbf{U}(t)=\alpha \int_{-\infty}^{t} \exp \left[-i \mathbf{M}\left(t-t^{\prime}\right)\right] \mathcal{F}_{\mathrm{NL}}(\mathbf{U})\left(t^{\prime}\right) d t^{\prime}+\mathbf{U}^{(0)}(t),
$$

where

$$
\mathbf{U}^{(0)}(t)=-\int_{-\infty}^{t} e^{-i \mathbf{M}\left(t-t^{\prime}\right)} \mathbf{J}\left(t^{\prime}\right) d t^{\prime} .
$$

An advantage of the integral form (2.12) is that it does not require the differentiability of $\mathbf{U}(t)$ with respect to time, allowing us to work in the space of continuous functions $\mathcal{C}\left((-\infty, T] ; \mathcal{H}^{s}\right)$ introduced above. Theorem 2.2 shows that the righthand side of $(2.12)$ is well defined for small $\mathbf{U} \in \mathcal{C}\left((-\infty, T] ; \mathcal{H}^{s}\right)$ (it belongs to $\mathcal{C}\left((-\infty, T] ; \mathcal{H}^{s-1}\right)$ since $\mathcal{F}_{\mathrm{NL}}(\mathbf{U})$ acts from $\mathcal{H}^{s}$ to $\left.\mathcal{H}^{s-1}\right)$

We would like to quote here a result from [6] (it will not be used in this paper and is presented to clarify the relations between different operators of interest).

THEOREM 2.3. Let $\alpha \leq \alpha_{0}$, an integer $s>d / 2$, let (1.7) hold, and for all $h, j=1, \ldots, h$,

$$
\begin{aligned}
& \int_{0}^{\infty} \cdots \int_{0}^{\infty}\left\|\mathbf{S}^{(h)}\left(\mathbf{r} ; t_{1}, t_{j-1}, 0, t_{j+1} \ldots, t_{h}\right)\right\|_{\mathbf{W B}_{2}^{s}\left(\mathbb{R}^{d}\right)} d t_{1} \cdots d t_{j-1} d t_{j+1} \cdots d t_{h} \\
& \leq C_{0 P} C_{1 P}^{h}
\end{aligned}
$$

and

$$
\int_{0}^{\infty} \cdots \int_{0}^{\infty}\left\|\frac{\partial}{\partial t_{j}} \mathbf{S}^{(h)}\left(\mathbf{r} ; t_{1}, \ldots, t_{h}\right)\right\|_{\mathbf{W B}_{2}^{s}\left(\mathbb{R}^{d}\right)} d t_{1} \cdots d t_{h} \leq C_{0 P} C_{1 P}^{h} .
$$

There exist constants $C_{U}, c_{0}>0$ such that the following statements hold. If $T \leq$ $c_{0} / \alpha$ and the excitation $\mathbf{J}$ is such that the function $\mathbf{U}^{0}$ defined by (2.13) satisfies the inequality

$$
\left\|\mathbf{U}^{0}\right\|_{\mathcal{C}\left((-\infty, T] ; \mathcal{H}^{s}\right)} \leq C_{U}
$$

then equation (2.12) has a unique solution $\mathbf{U}$ in $\mathcal{C}\left((-\infty, T] ; \mathcal{H}^{s}\right)$. Moreover, for $T \leq c_{0} / \alpha$

$$
\left\|\mathbf{U}-\mathbf{U}^{0}-\alpha \mathbf{U}^{(1)}(t)\right\|_{\mathcal{C}\left((-\infty, T] ; \mathcal{H}^{s}\right)} \leq \alpha^{2} T C_{U 2}
$$


Here $\mathbf{U}^{0}(t)$ is given by (2.13) and the first nonlinear response $\mathbf{U}^{(1)}(t)$ is given by

$$
\mathbf{U}^{(1)}(t)=\int_{-\infty}^{t} \exp \left[-i \mathbf{M}\left(t-t^{\prime}\right)\right] \mathcal{F}_{N L}^{\left(h_{0}\right)}\left(\mathbf{U}^{(0)}\right)\left(t^{\prime}\right) d t^{\prime}
$$

where

$$
\mathcal{F}_{N L}^{\left(h_{0}\right)}(\mathbf{U})=\left[\begin{array}{c}
\mathbf{0} \\
\nabla \times \mathcal{S}^{\left(h_{0}\right)}(\mathbf{r}, t ; \mathbf{D})
\end{array}\right], \mathbf{U}=\left[\begin{array}{l}
\mathbf{D} \\
\mathbf{B}
\end{array}\right]
$$

Everywhere we assume that $h_{0} \geq 2$. According to Theorem 2.3,

$$
\mathbf{U}(t)=\mathbf{U}^{0}(t)+\alpha \mathbf{U}^{(1)}(t)+O\left(\alpha^{2}\right)
$$

for $0 \leq t \leq c_{0} / \alpha$, where $\mathbf{U}^{0}(\mathbf{r}, t)$ is given by (2.13) and the first nonlinear response is given by (2.17).

An alternative to description (1.5) of the polarization, which is used more often in physical literature, is provided by the frequency response tensor $\chi^{(h)}$, known as the nonlinear susceptibility; see, for example, [11], [12]:

$$
\begin{aligned}
\chi_{\mathbf{S}}^{(h)} & (\mathbf{r} ; \vec{\omega} ; \overrightarrow{\mathbf{D}}(\mathbf{r})) \\
& =\int_{-\infty}^{\infty} \cdots \int_{-\infty}^{\infty} e^{\left(i \sum_{j=1}^{h} \omega_{j} t_{j}\right)} \mathbf{S}_{0}^{(h)}\left(\mathbf{r}, t_{1}, \ldots, t_{h}\right) \prod_{j=1}^{h} \mathbf{D}_{j}(\mathbf{r}) \prod_{j=1}^{h} d t_{j} .
\end{aligned}
$$

We call this tensor the susceptibility symbol. It is just the Fourier transform of the kernel $\mathbf{S}_{0}^{(h)}$. Similarly, the susceptibility symbol $\chi_{\mathbf{F}}^{(h)}$ corresponding to $\mathcal{F}_{\mathrm{NL}}^{\left(h_{0}\right)}(\mathbf{U})$ in (2.18) is defined by

$$
\chi_{\mathbf{F}}^{(h)}(\mathbf{r} ; \vec{\omega} ; \overrightarrow{\mathbf{U}}(\mathbf{r}))=\left[\begin{array}{c}
\mathbf{0} \\
\nabla \times \chi_{\mathbf{S}}^{(h)}(\mathbf{r} ; \vec{\omega} ; \overrightarrow{\mathbf{D}}(\mathbf{r}))
\end{array}\right], \mathbf{U}=\left[\begin{array}{c}
\mathbf{D} \\
\mathbf{B}
\end{array}\right] .
$$

Both $\chi_{\mathbf{S}}^{(h)}(\mathbf{r} ; \vec{\omega} ; \cdot)$ and $\chi_{\mathbf{F}}^{(h)}(\mathbf{r} ; \vec{\omega} ; \cdot)$ are multilinear operators that depend on $\vec{\omega}$ as a parameter. The operator $\mathcal{F}_{\mathrm{NL}}^{\left(h_{0}\right)}(\mathbf{U})$ acts from the space $\mathcal{H}^{s}$ into $\mathcal{H}^{s-1}$ for every $\vec{\omega}$. One of the goals of this paper is to show that the susceptibility $\chi_{\mathbf{S}}^{(h)}$ can be used to define an operator $\mathcal{K}_{\chi_{\mathbf{S}}^{(h)}}$ and to show that this operator is a good approximation to the operator $\mathcal{S}^{\left(h_{0}\right)}\left(\mathbf{U}^{(0)}\right)$ in $(2.17),(2.18)$. Or, equivalently, $\chi_{\mathbf{F}}^{(h)}(\mathbf{r} ; \vec{\omega} ; \cdot)$ can be used to define an operator $\mathcal{K}_{\chi_{\mathrm{F}}^{(h)}}$ and to show that this operator is a good approximation to the operator $\mathcal{F}_{\mathrm{NL}}^{\left(\chi_{0}\right)}\left(\mathbf{U}^{0}\right)$ in $(2.17),(2.18)$. The above relations can be used to approximate integrals of the form (2.17), (2.13) by integrals involving the susceptibilities. The important special case of periodic structures where the spectral representation is more explicit, is briefly discussed in the last section (see [5] for a detailed discussion).

\section{Abstract wavepackets}

Let $\mathcal{H}$ be a Hilbert space $\mathcal{H}$, and $\mathbf{M}$ a selfadjoint (unbounded) operator in it. In this section $\mathbf{M}$ is a general selfadjoint operator in a Hilbert space $\mathcal{H}$ rather than the Maxwell operator considered in the previous section. Let us consider also equation (2.1) with its formal solution given by (2.13). 
We denote by $\mathcal{E}(\omega)$ the spectral resolution of identity associated with the selfadjoint operator $\mathbf{M}$ which is a family of orthogonal projections in $\mathcal{H},[\mathbf{2 4}]$. In particular,

$$
\begin{gathered}
\mathcal{E}(\omega) u=\int_{-\infty}^{\omega} d \mathcal{E}\left(\omega^{\prime}\right) u \\
\lim _{\omega \rightarrow-\infty} \mathcal{E}(\omega) u=0, \lim _{\omega \rightarrow+\infty} \mathcal{E}(\omega) u=u, u \in \mathcal{H} .
\end{gathered}
$$

In addition,

$$
u=\int_{-\infty}^{\infty} d \mathcal{E}(\omega) u, \quad \mathbf{M} u=\int_{-\infty}^{\infty} \omega d \mathcal{E}(\omega) u
$$

and for a function $f(\mathbf{M})$ of the operator $\mathbf{M}$,

$$
f(\mathbf{M}) u=\int_{-\infty}^{\infty} f(\omega) d \mathcal{E}(\omega) u .
$$

In particular,

$$
\exp [-i \mathbf{M} t] u=\int_{-\infty}^{\infty} \exp [-i \omega t] d \mathcal{E}(\omega) u .
$$

We introduce the Hilbert space $\mathcal{H}^{s}$ with the following finite norm:

$$
\|u\|_{\mathcal{H}^{s}}^{2}=\int_{-\infty}^{\infty}\left(1+|\omega|^{2}\right)^{s} d(\mathcal{E}(\omega) u, u)_{\mathcal{H}} .
$$

Then we have

$$
\|f(\mathbf{M}) u\|_{\mathcal{H}^{s}}^{2}=\int_{-\infty}^{\infty}|f(\omega)|^{2}\left(1+|\omega|^{2}\right)^{s} d(\mathcal{E}(\omega) u, u)_{\mathcal{H}} .
$$

Let $\left\{\omega_{i}\right\}_{1}^{\infty}$ be a monotone sequence,

$$
\omega_{1}<\cdots<\omega_{i}<\omega_{i+1}<\cdots .
$$

Note that $\left(\mathcal{E}\left(\omega_{i}\right)-\mathcal{E}\left(\omega_{i-1}\right)\right) u$ are orthogonal to $\left(\mathcal{E}\left(\omega_{j}\right)-\mathcal{E}\left(\omega_{j-1}\right)\right) u$ in $\mathcal{H}^{s}$ when $i \neq j$. Hence, for any bounded sequence of numbers $a_{i}$ and $\left\{\omega_{i}\right\}_{1}^{\infty}$ we have

$$
\begin{aligned}
\sum_{i=1}^{\infty} & \left\|a_{i}\left(\mathcal{E}\left(\omega_{i}\right)-\mathcal{E}\left(\omega_{i-1}\right)\right) u\right\|_{\mathcal{H}^{s}}^{2} \\
& =\sum_{i=1}^{\infty}\left|a_{i}\right|^{2}\left\|\left(\mathcal{E}\left(\omega_{i}\right)-\mathcal{E}\left(\omega_{i-1}\right)\right) u\right\|_{\mathcal{H}^{s}}^{2} \leq\|u\|_{\mathcal{H}^{s}}^{2} \sup _{i}\left|a_{i}\right|^{2}, \\
& \left\|\sum_{i=1}^{\infty} a_{i}\left(\mathcal{E}\left(\omega_{i}\right)-\mathcal{E}\left(\omega_{i-1}\right)\right) u\right\|_{\mathcal{H}^{s}} \leq\|u\|_{\mathcal{H}^{s}} \sup _{i}\left|a_{i}\right| .
\end{aligned}
$$

To define a wavepacket excitation, we take an integrable $\mathcal{H}^{s}$-valued function $\mathbf{j}^{0}(\tau)$ of a real variable $\tau$ such that

$$
\begin{gathered}
\mathbf{j}^{0}(\tau)=0 \text { for } \tau \leq 0, \\
\left\|\mathbf{j}^{0}(\tau)\right\|_{\mathcal{H}^{s}} \leq \varrho C_{\mathbf{j}} \text { for all } \tau .
\end{gathered}
$$

We set $\tau=\varrho t$, where $\varrho$ is a small parameter, and define the wavepacket excitations $\mathbf{J}$ in the form

$$
\mathbf{J}(t, \tau)=\int_{-\infty}^{\infty} \exp [-i \omega t] d \mathcal{E}(\omega) \mathbf{j}^{0}(\tau) .
$$


Using (3.4), we get

$$
\mathbf{J}(t, \tau)=\exp [-i \mathbf{M} t] \mathbf{j}^{0}(\tau) .
$$

By (3.6), and since $\exp [-i \mathbf{M} t]$ is a unitary operator, $\mathbf{J}(t, \tau)$ is bounded,

$$
\|\mathbf{J}(t, \tau)\|_{\mathcal{H}^{s}} \leq \varrho C_{\mathbf{j}}
$$

Having in mind (2.13), we define $\mathbf{U}^{0}$ by

$$
\begin{aligned}
\mathbf{U}^{0}(t) & =-\int_{-\infty}^{t} \exp \left[-i \mathbf{M}\left(t-t^{\prime}\right)\right] \mathbf{J}\left(t^{\prime}, \varrho t^{\prime}\right) d t^{\prime} \\
& =-\int_{-\infty}^{t} \int_{-\infty}^{\infty} \exp \left[-i \omega\left(t-t^{\prime}\right)\right] \exp \left[-i \omega t^{\prime}\right] d \mathcal{E}(\omega) \mathbf{j}^{0}\left(\varrho t^{\prime}\right) d t^{\prime} \\
& =-\int_{-\infty}^{\infty} \exp [-i \omega t] d \mathcal{E}(\omega) \int_{-\infty}^{t} \mathbf{j}^{0}\left(\varrho t^{\prime}\right) d t^{\prime} .
\end{aligned}
$$

Obviously, $\mathbf{U}^{0}(t)$ is a solution of (2.1) with $\mathbf{J}=\mathbf{J}(t, \tau)$. Then we introduce

$$
\mathbf{v}(\tau)=-\varrho^{-1} \int_{-\infty}^{\tau} \mathbf{j}^{0}\left(\tau^{\prime}\right) d \tau^{\prime}
$$

and obtain

$$
\mathbf{U}^{0}(t, \tau)=\int_{-\infty}^{\infty} \exp [-i \omega t] d \mathcal{E}(\omega) \mathbf{v}(\tau), \quad \tau=\varrho t,
$$

or, in the operator form,

$$
\mathbf{U}^{0}(t, \tau)=\exp [-i \mathbf{M} t] \mathbf{v}(\tau) .
$$

Here $\mathbf{v}(\tau)$ can be viewed as a slow amplitude of the wavepacket $\mathbf{U}^{0}(t, \tau)$. By (3.15)

$$
\left\|\mathbf{U}^{0}(t, \tau)\right\|_{\mathcal{H}^{s}} \leq T C_{\mathbf{j}}, \quad-\infty<\tau \leq T .
$$

\section{The action of multilinear operators with time dispersion on wavepackets}

In this section we consider $h$-tuples $\overrightarrow{\mathbf{u}}=\left(\mathbf{u}_{1}, \ldots, \mathbf{u}_{h}\right)$ of vector-valued functions $\mathbf{u} \in \mathcal{H}^{s}$ that have the form of wavepackets (3.18):

$$
\mathbf{u}_{l}(t, \tau)=\int_{-\infty}^{\infty} \exp [-i \omega t] d \mathcal{E}(\omega) \mathbf{v}_{l}(\tau), \quad \tau=\varrho t, l=1, \ldots, h .
$$

We consider integral operators with time dispersion that act on $\overrightarrow{\mathbf{u}}(t, \varrho t)$ and are similar to (1.5):

$$
\begin{aligned}
& \mathcal{K}(\overrightarrow{\mathbf{u}} ; t) \\
= & \int_{-\infty}^{t} \ldots \int_{-\infty}^{t} \mathbf{K}^{(h)}\left(t-t_{1}, \ldots, t-t_{h} ; \mathbf{u}_{1}\left(t_{1}, \varrho t_{1}\right), \ldots, \mathbf{u}_{h}\left(t_{h}, \varrho t_{h}\right)\right) d t_{1} \cdots d t_{h},
\end{aligned}
$$

where $\mathbf{K}^{(h)}\left(t_{1}, \ldots, t_{h}\right)$ is a bounded $h$-linear operator from $\mathcal{H}^{s}$ to $\mathcal{H}^{s-q}$ for every $t_{1}, \ldots, t_{h} \geq 0$. We assume that the following condition holds:

$$
\int_{0}^{\infty} \cdots \int_{0}^{\infty} \sum_{l}\left\|\mathbf{K}^{(h)}\left(t_{1}, \ldots, t_{h}\right)\right\|_{\mathcal{H}^{s}, \mathcal{H}^{s-q}} d t_{1} \cdots d t_{h} \leq C_{s 0 \mathbf{K}}
$$


We introduce then an operator $\overline{\mathcal{K}}$,

$$
\begin{aligned}
& \overline{\mathcal{K}}(\overrightarrow{\mathbf{u}}, t) \\
= & \int_{-\infty}^{t} \cdots \int_{-\infty}^{t} \mathbf{K}^{(h)}\left(t-t_{1}, \ldots, t-t_{h} ; \mathbf{u}_{1}\left(t_{1}, \varrho t\right), \ldots, \mathbf{u}_{h}\left(t_{h}, \varrho t\right)\right) d t_{1} \cdots d t_{h}
\end{aligned}
$$

where $\mathbf{u}_{l}(t, \tau)$ are given in (4.1). This operator will be used later to define the susceptibilities.

The next lemma shows that $\mathcal{K}(\overrightarrow{\mathbf{u}}, t)$ and $\overline{\mathcal{K}}(\overrightarrow{\mathbf{u}}, t)$ are close for small $\varrho$.

Lemma 4.1. Let $-\infty<t \leq T / \varrho,-\infty<\tau \leq T$. Let $\mathbf{u}_{1}\left(t_{1}, \tau\right), \ldots, \mathbf{u}_{h}\left(t_{h}, \tau\right)$ be bounded,

$$
\sum_{l=1}^{h} \sup _{t \leq T}\left\|\mathbf{u}_{l}(t, \tau)\right\|_{\mathcal{H}^{s}} \leq C_{\mathbf{u}},
$$

and satisfy the Lipschitz condition

$$
\sum_{l}\left\|\mathbf{u}_{l}\left(t_{l}, \tau\right)-\mathbf{u}_{l}\left(t_{l}, \tau^{\prime}\right)\right\|_{\mathcal{H}^{s}} \leq L_{\mathbf{u}}\left|\tau-\tau^{\prime}\right|
$$

Assume also that

$$
\int_{0}^{\infty} \cdots \int_{0}^{\infty} \sum_{l}\left|t_{l}\right|\left\|\mathbf{K}^{(h)}\left(t_{1}, \ldots, t_{h}\right)\right\|_{\mathcal{H}^{s}, \mathcal{H}^{s-q}} d t_{1} \cdots d t_{h} \leq C_{s 1 \mathbf{K}} .
$$

Then

$$
\sup _{t \leq T}\|\mathcal{K}(\overrightarrow{\mathbf{u}}, t)-\overline{\mathcal{K}}(\overrightarrow{\mathbf{u}}, t)\|_{\mathcal{H}^{s}} \leq \varrho h C_{\mathbf{u}}^{h-1} L_{\mathbf{u}} C_{s 1 \mathbf{K}} .
$$

Proof. If $\mathcal{A}$ is an $h$-linear operator, then

$$
\begin{aligned}
\mathcal{A}\left(\mathbf{v}_{1}, \ldots, \mathbf{v}_{h}\right)-\mathcal{A}\left(\mathbf{v}_{1}^{\prime}, \ldots, \mathbf{v}_{h}^{\prime}\right) & \\
= & \mathcal{A}\left(\mathbf{v}_{1}-\mathbf{v}_{1}^{\prime}, \mathbf{v}_{2}, \ldots, \mathbf{v}_{h}\right)+\cdots+\mathcal{A}\left(\mathbf{v}_{1}^{\prime}, \ldots, \mathbf{v}_{l-1}^{\prime}, \mathbf{v}_{l}-\mathbf{v}_{l}^{\prime}, \mathbf{v}_{l+1} \ldots, \mathbf{v}_{h}\right) \\
& +\cdots+\mathcal{A}\left(\mathbf{v}_{1}^{\prime}, \ldots, \mathbf{v}_{h-1}^{\prime}, \mathbf{v}_{h}-\mathbf{v}_{h}^{\prime}\right)
\end{aligned}
$$

Using this identity with $\mathcal{A}=\mathbf{K}^{(h)}\left(t-t_{1}, \ldots, t-t_{h}\right)$ we get

$$
\begin{aligned}
\|\mathcal{K}(\overrightarrow{\mathbf{u}}, t)-\overline{\mathcal{K}}(\overrightarrow{\mathbf{u}}, t)\|_{\mathcal{H}^{s}} \\
\leq \int_{-\infty}^{t} \cdots \int_{-\infty}^{t}\left\|\mathbf{K}^{(h)}\left(t-t_{1}, \ldots, t-t_{h}\right)\right\|_{\mathcal{H}^{s}, \mathcal{H}^{s-q}} \\
\quad \sum_{l}\left\|\mathbf{u}_{l}\left(t_{l}, \tau\right)-\mathbf{u}_{l}\left(t_{l}, \tau^{\prime}\right)\right\|_{\mathcal{H}^{s}} \prod_{j<l}\left\|\mathbf{u}_{j}\left(t_{j}, \tau\right)\right\| \prod_{j>l}\left\|\mathbf{u}_{j}\left(t_{j}, \tau^{\prime}\right)\right\| d t_{1} \cdots d t_{h} \\
\leq \varrho C_{\mathbf{u}}^{h-1} L_{\mathbf{u}} \int_{-\infty}^{t} \cdots \int_{-\infty}^{t}\left\|\mathbf{K}^{(h)}\left(t-t_{1}, \ldots, t-t_{h}\right)\right\|_{\mathcal{H}^{s}, \mathcal{H}^{s-q}} \sum_{l}\left|t-t_{l}\right| d t_{1} \cdots d t_{h} \\
\leq \varrho C_{\mathbf{u}}^{h-1} L_{\mathbf{u}} C_{s 1 \mathbf{K}} .
\end{aligned}
$$


REMARK 4.2. One can similarly get an approximation of a higher order in $\varrho$. By Taylor's formula

$$
\begin{aligned}
\mathbf{u}_{l}\left(t_{l}, \varrho t_{l}\right)= & \mathbf{u}_{l}\left(t_{l}, \varrho t+\varrho\left(\left(t_{l}-t\right)\right)\right) \\
= & \mathbf{u}_{l}\left(t_{l}, \varrho t\right) \\
& +\sum_{j=0}^{N-1} \frac{1}{j !} \varrho^{j}\left(t_{l}-t\right)^{j} \frac{\partial^{j}}{\partial \tau^{j}} \mathbf{u}\left(t_{l}, \varrho t\right)+O\left(\varrho\left|t-t_{l}\right|^{N}\left\|\partial_{\tau}^{N} \mathbf{u}\right\|\right) .
\end{aligned}
$$

Plugging in the Taylor polynomials from (4.10) into (4.2), we collect terms with equal powers of $\prod_{l}\left(t_{l}-t\right)^{j_{l}}$. One obtains a sum of expressions of the form

$$
\begin{aligned}
& \overline{\mathcal{K}}_{p}(\overrightarrow{\mathbf{u}}, t) \\
& =\varrho^{p} \sum_{l_{1}+\cdots+l_{h}=p} \int \cdots \int \mathbf{K}^{(h)}\left(t-t_{1}, \ldots, t-t_{h} ; \frac{\partial^{l_{1}}}{\partial \tau^{l_{1}}} \mathbf{u}\left(t_{1}, \varrho t\right), \ldots, \frac{\partial^{l_{h}}}{\partial \tau^{l_{h}}} \mathbf{u}\left(t_{h}, \varrho t\right)\right) \\
& \quad \times \prod_{j=1}^{h} \frac{\left(t_{l}-t\right)^{l_{j}}}{l_{j} !} d t_{1} \cdots d t_{h} .
\end{aligned}
$$

If for some $N \geq 1$

$$
\int\left|\mathbf{K}^{(h)}\left(\mathbf{r} ; t-t_{1}, \ldots, t-t_{h}\right)\right| \sum\left|t-t_{l}\right|^{N} d t \leq C
$$

and

$$
\left\|\frac{\partial^{n}}{\partial \tau^{n}} \mathbf{u}\left(t_{l}, \tau\right)\right\| \mathcal{H}^{s} \leq C_{N}(\mathbf{u}) \text { for all } \tau \leq T, n \leq N,
$$

then, similarly to Lemma 4.1 , we obtain that

$$
\sup _{t \leq T}\left\|\mathcal{K}(\overrightarrow{\mathbf{u}}, t)-\sum_{p=0}^{N-1} \mathcal{K}_{p, \chi}(\overrightarrow{\mathbf{u}}, t)\right\|_{\mathcal{H}^{s}} \leq C_{N}^{\prime} \varrho^{N} .
$$

Now we consider the action of the operator $\overline{\mathcal{K}}$ on wavepackets $\overrightarrow{\mathbf{u}}(t, \tau)$. The operator $\overline{\mathcal{K}}(\overrightarrow{\mathbf{u}}, t)$ can be expressed in terms of the slow amplitudes $\overrightarrow{\mathbf{v}}$ using (4.1) in the operator form $(3.19)$,

$$
\begin{aligned}
\overline{\mathcal{K}}(\overrightarrow{\mathbf{u}}, t)= & \int_{-\infty}^{t}, \ldots \int_{-\infty}^{t} d t_{1} \cdots d t_{h} \\
& \times \mathbf{K}^{(h)}\left(t-t_{1}, \ldots, t-t_{h} ; e^{\left[-i \mathbf{M} t_{1}\right]} \mathbf{v}_{1}(\varrho t), \ldots, e^{\left[-i \mathbf{M} t_{h}\right]} \mathbf{v}_{h}(\varrho t)\right) .
\end{aligned}
$$

According to (4.1),

$$
e^{[-i \mathbf{M} t]} \mathbf{v}_{l}(\tau)=\mathbf{u}_{l}(t, \tau)
$$

Using the notation $t-t_{l}=z_{l}$ we obtain

$$
e^{\left[-i \mathbf{M} t_{l}\right]} \mathbf{v}_{l}(\tau)=e^{\left[i \mathbf{M} z_{l}\right]} \mathbf{u}_{l}(t, \tau) .
$$

Then we rewrite (4.14):

$$
\begin{aligned}
\overline{\mathcal{K}}(\overrightarrow{\mathbf{u}}, t)= & \int_{0}^{\infty} \cdots \int_{0}^{\infty} d z_{1} \cdots d z_{h} \\
& \times \mathbf{K}^{(h)}\left(z_{1}, \ldots, z_{h} ; e^{\left[i \mathbf{M} z_{1}\right]} \mathbf{u}_{1}(\tau), \ldots, e^{\left[i \mathbf{M} z_{h}\right]} \mathbf{u}_{h}(\tau)\right),
\end{aligned}
$$


where $\mathbf{u}_{l}=\mathbf{u}_{l}(t, \tau)$ do not depend on $z_{l}$. To write this expression in terms of spectral projections we need to develop appropriate notation, which is done in the following section.

\section{Multilinear operators and spectral decomposition}

In this section we define integrals of the form (0.6) for sufficiently general classes of frequency dependent multilinear operators $\chi^{(h)}(\omega)$. We consider two cases of $\omega$ dependence. In the first case $\chi^{(h)}$ has coefficients depending on the product of functions $f\left(\omega_{l}\right)$ of one variable, or on a sum of such products. In the second case $\chi^{(h)}(\omega)$ is defined in terms of the Fourier transform.

Let $\mathcal{E}(\omega)$ be the spectral family of projections corresponding to a selfadjoint operator $\mathbf{M}$, and let $\mathcal{H}^{s}$ be the domain of $\mathbf{M}^{s}$. Let $\chi\left(\omega_{1}, \ldots, \omega_{h} ; \mathbf{u}_{1}, \ldots, \mathbf{u}_{h}\right)$ be a continuous $h$-linear operator from $\left(\mathcal{H}^{s}\right)^{h}$ into $\mathcal{H}^{s-q}$ which continuously depends on the parameters $\omega_{1}, \ldots, \omega_{h}$ and $\mathbf{u}_{1}, \ldots, \mathbf{u}_{h} \in \mathcal{H}^{s}$. We first pick two finite numbers $-\omega_{l}^{-}<\omega_{l}^{+}$and then subdivide the interval $\left[-\omega_{l}^{-}, \omega_{l}^{+}\right]$into subintervals $\left[\omega_{l, i_{l}-1}, \omega_{l, i_{l}}\right]$ for every $l=1, \ldots, h$, introducing

$$
\bar{d}=\sup _{l, i_{l}}\left|\omega_{l, i_{l}}-\omega_{l, i_{l}-1}\right| .
$$

It is assumed that $\omega_{l, i}$ are monotonically increasing in $i$. Let us consider RiemannStijelties integral sums

$$
\sum_{i_{1}, \ldots, i_{h}} \chi\left(\omega_{1, i_{1}}^{\prime}, \ldots, \omega_{h, i_{h}}^{\prime} ; \Delta_{i_{1}} \mathcal{E}(\omega) \mathbf{u}_{1}, \ldots, \Delta_{i_{h}} \mathcal{E}(\omega) \mathbf{u}_{h}\right)
$$

where

$$
\Delta_{i_{l}} \mathcal{E}(\omega) \mathbf{u}_{l}=\left[\mathcal{E}\left(\omega_{1, i_{l}}\right)-\mathcal{E}\left(\omega_{1, i_{l}-1}\right)\right] \mathbf{u}_{l}, l=1, \ldots, h,
$$

and $\omega_{1, i_{l}}^{\prime} \in\left[\omega_{l, i_{l}-1}, \omega_{l, i_{l}}\right]$. We now define the integral

$$
\int_{-\omega_{1}^{-}}^{\omega_{1}^{+}} \ldots \int_{-\omega_{h}^{-}}^{\omega_{h}^{+}} \chi\left(\omega_{1}^{\prime}, \ldots, \omega_{h}^{\prime} ; d \mathcal{E}(\omega) \mathbf{u}_{1}, \ldots, d \mathcal{E}(\omega) \mathbf{u}_{h}\right)
$$

as a limit of the Riemann-Stijelties sums (5.1) as $\bar{d} \rightarrow 0$. After that we let $-\omega_{l}^{-} \rightarrow$ $-\infty, \omega_{l}^{+} \rightarrow \infty, l=1, \ldots, h$. Having that done we continue on with the following definition.

Definition 5.1. Let $\mathbf{M}$ be a selfadjoint operator in $\mathcal{H}$, and let $\chi\left(\omega_{1}, \ldots, \omega_{h}\right.$; $\left.\mathbf{u}_{1}, \ldots, \mathbf{u}_{h}\right)$ be a bounded $h$-linear operator from $\left(\mathcal{H}^{s}\right)^{h}$ into $\mathcal{H}^{s-q}$ that acts on $\mathbf{u}_{1}, \ldots, \mathbf{u}_{h} \in \mathcal{H}^{s}$ and is continuous in the parameters $\omega_{1}, \ldots, \omega_{h}$. Assume that the following limit exists in $\mathcal{H}^{s-q}$ for any $\mathbf{u}_{1}, \ldots, \mathbf{u}_{h} \in \mathcal{H}^{s}$ :

$$
\begin{aligned}
& \mathcal{A}_{\mathbf{M}, \chi}\left(\mathbf{u}_{1}, \ldots, \mathbf{u}_{h}\right) \\
& =\lim _{\substack{\omega_{l}^{-} \rightarrow \infty, \omega_{l}^{+} \rightarrow \infty \\
l=1, \ldots, h}} \lim _{\bar{d} \rightarrow 0} \sum_{i_{1}, \ldots, i_{h}} \chi\left(\omega_{1, i_{1}}^{\prime}, \ldots, \omega_{h, i_{h}}^{\prime} ; \Delta_{i_{1}} \mathcal{E}(\omega) \mathbf{u}_{1}, \ldots, \Delta_{i_{h}} \mathcal{E}(\omega) \mathbf{u}_{h}\right) .
\end{aligned}
$$

Then we set

$$
\mathcal{A}_{M, \chi}\left(\mathbf{u}_{1}, \ldots, \mathbf{u}_{h}\right)=\int_{-\infty}^{\infty} \cdots \int_{-\infty}^{\infty} \chi\left(\omega_{1}, \ldots, \omega_{h} ; d \mathcal{E}\left(\omega_{1}\right) \mathbf{u}_{1}, \ldots, d \mathcal{E}\left(\omega_{h}\right) \mathbf{u}_{h}\right)
$$


Clearly, if $\mathcal{A}_{M, \chi}\left(\mathbf{u}_{1}, \ldots, \mathbf{u}_{h}\right)$ exists, it is an $h$-linear operator with respect to $\mathbf{u}_{1}, \ldots, \mathbf{u}_{h}$.

The following statement gives a simple sufficient condition for the existence of an integral of the form (5.5) in the case of

$$
\chi\left(\omega_{1}, \ldots, \omega_{h} ; \mathbf{u}_{1}, \ldots, \mathbf{u}_{h}\right)=f_{1}\left(\omega_{1}\right) \cdots f_{h}\left(\omega_{h}\right) \mathcal{A}_{0}\left(\mathbf{u}_{1}, \ldots, \mathbf{u}_{h}\right) .
$$

TheOREm 5.2. Let $\mathcal{A}_{0}$ be a continuous h-linear operator from $\left(\mathcal{H}^{s}\right)^{h}$ into $\mathcal{H}^{s-q}$, and let $f_{i}(\omega), i=1, \ldots, h$, be bounded complex-valued continuous functions of a real variable $\omega$. Then for any $\mathbf{u}_{1}, \ldots, \mathbf{u}_{h} \in \mathcal{H}^{s}$,

$$
\begin{aligned}
\int_{-\infty}^{\infty} & \cdots \int_{-\infty}^{\infty} f_{1}\left(\omega_{1}\right) \cdots f_{h}\left(\omega_{h}\right) \mathcal{A}_{0}\left(d \mathcal{E}\left(\omega_{1}\right) \mathbf{u}_{1}, \ldots, d \mathcal{E}\left(\omega_{h}\right) \mathbf{u}_{h}\right) \\
& =\mathcal{A}_{0}\left(f_{1}(\mathbf{M}) \mathbf{u}_{1}, \ldots, f_{h}(\mathbf{M}) \mathbf{u}_{h}\right)
\end{aligned}
$$

Proof. We pick $\left\|\mathbf{u}_{1}\right\|_{\mathcal{H}^{s}} \leq 1, \ldots,\left\|\mathbf{u}_{h}\right\|_{\mathcal{H}^{s}} \leq 1$. Then the vector

$$
g=\mathcal{A}_{0}\left(f_{1}(\mathbf{M}) \mathbf{u}_{1}, \ldots, f_{h}(\mathbf{M}) \mathbf{u}_{h}\right) \in \mathcal{H}^{s}
$$

is well defined and depends continuously on $\mathbf{u}_{1}, \ldots, \mathbf{u}_{h}$ since the linear operators $f_{i}(\mathbf{M})$ are bounded in $\mathcal{H}^{s}$. In addition, we have $f_{l}(\mathbf{M}) \mathbf{u}_{l}=\int f_{l}\left(\omega_{l}\right) d \mathcal{E}\left(\omega_{l}\right) \mathbf{u}_{l}$.

Let us pick any $0<\epsilon_{0} \leq 1$. By (3.2) we can always take $\omega_{l}^{-}$and $\omega_{l}^{+}$large enough that

$$
\int_{-\infty}^{-\omega_{l}^{-}} d\left(\mathcal{E}\left(\omega_{l}\right) \mathbf{u}_{l}, \mathbf{u}_{l}\right)_{\mathcal{H}^{s}} \leq \epsilon_{0}^{2}, \quad \int_{\omega_{l}^{+}}^{\infty} d\left(\mathcal{E}\left(\omega_{l}\right) \mathbf{u}_{l}, \mathbf{u}_{l}\right)_{\mathcal{H}^{s}} \leq \epsilon_{0}^{2}
$$

for every $l=1, \ldots, h$. Now if

$$
\mathbf{w}_{l}=\left[\mathcal{E}\left(\omega_{l}^{+}\right)-\mathcal{E}\left(-\omega_{l}^{-}\right)\right] \mathbf{u}_{l}
$$

then obviously

$$
\left\|\mathbf{w}_{l}-\mathbf{u}_{l}\right\|_{\mathcal{H}^{s}}^{2} \leq 2 \epsilon_{0}^{2}
$$

Since $f_{l}(\omega)$ are bounded, $\left|f_{l}(\omega)\right| \leq \bar{f}$, we get

$$
\left\|f_{l}(\mathbf{M}) \mathbf{w}_{l}-f_{l}(\mathbf{M}) \mathbf{u}_{l}\right\|_{\mathcal{H}^{s}}^{2} \leq 2 \epsilon_{0}^{2} \bar{f}^{2}
$$

Let us take $\left|\omega_{l, i_{l}}-\omega_{l, i_{l}-1}\right| \leq \delta$, where $\delta$ is small enough that the variation of the function $f_{l}\left(\omega_{l}\right)$ on every interval $a \leq \omega_{l} \leq a+\delta$ is less than a given $\epsilon$ for every $l=1, \ldots, h$ when $-\omega_{l}^{-} \leq a \leq \omega_{l}^{+}$. We then subdivide $\left[\omega_{l}^{-}, \omega_{l}^{+}\right]$into intervals of length at most $\delta$, take $\omega_{l, i_{l}}^{\prime}$ in every interval, and introduce

$$
\mathbf{u}_{l}^{\prime}=\sum_{i_{l}} f_{l}\left(\omega_{l, i_{l}}^{\prime}\right) \Delta_{i_{l}} \mathcal{E}(\omega) \mathbf{u}_{l}
$$

where $\Delta_{i_{l}} \mathcal{E}(\omega)$ is defined in $(5.2), \omega_{l, i_{l}}^{\prime} \in\left[\omega_{l, i_{l}-1}, \omega_{l, i_{l}}\right]$. By (3.10) and (3.7)

$$
\begin{aligned}
& \left\|f_{l}(\mathbf{M}) \mathbf{w}_{l}-\mathbf{u}_{l}^{\prime}\right\|_{\mathcal{H}^{s}}^{2} \\
& \quad \leq \sum_{i_{l}} \int_{\omega_{l, i_{l}-1}}^{\omega_{l, i_{l}}}\left|f_{l}\left(\omega_{l}\right)-f_{l}\left(\omega_{l, i_{l}}^{\prime}\right)\right|^{2} d\left(\mathcal{E}\left(\omega_{l}\right) \mathbf{u}_{l}, \mathbf{u}_{l}\right)_{\mathcal{H}^{s}} \leq \epsilon^{2}\left\|\mathbf{u}_{l}\right\|_{\mathcal{H}^{s}}^{2} .
\end{aligned}
$$

From (3.10) we deduce that for $\left|\omega_{l}-\omega_{l, i_{l}}^{\prime}\right| \leq \delta$ we get

$$
\left\|\sum_{i_{l}}\left(f_{l}\left(\omega_{l}\right)-f_{l}\left(\omega_{l, i_{l}}^{\prime}\right)\right) \Delta_{i_{l}} \mathcal{E}(\omega) \mathbf{u}_{l}\right\|_{\mathcal{H}^{s}} \leq \epsilon\left\|\mathbf{u}_{l}\right\|_{\mathcal{H}^{s}} .
$$


Consequently,

$$
\left\|f_{l}(\mathbf{M}) \mathbf{w}_{l}-\mathbf{u}_{l}^{\prime}\right\|_{\mathcal{H}^{s}} \leq \epsilon\left\|\mathbf{u}_{l}\right\|_{\mathcal{H}^{s}} .
$$

Let us consider

$$
g^{\prime}=\sum_{i_{1}, \ldots, i_{h}} f_{1}\left(\omega_{1, i_{1}}^{\prime}\right) \cdots f_{h}\left(\omega_{h, i_{h}}^{\prime}\right) \mathcal{A}_{0}\left(\Delta_{i_{1}} \mathcal{E}(\omega) \mathbf{u}_{1}, \ldots, \Delta_{i_{h}} \mathcal{E}(\omega) \mathbf{u}_{h}\right),
$$

which is an integral sum of (5.3). Clearly, $g^{\prime}=\mathcal{A}_{0}\left(\mathbf{u}_{1}^{\prime}, \ldots, \mathbf{u}_{h}^{\prime}\right)$. By (5.12) and (4.9), since $\left|f_{l}(\omega)\right| \leq \bar{f}$,

$$
\begin{aligned}
& \left\|\mathcal{A}_{0}\left(f_{1}(\mathbf{M}) \mathbf{w}_{1}, \ldots, f_{h}(\mathbf{M}) \mathbf{w}_{h}\right)-\mathcal{A}_{0}\left(\mathbf{u}_{1}^{\prime}, \ldots, \mathbf{u}_{h}^{\prime}\right)\right\|_{\mathcal{H}^{s-q}} \\
& \leq \epsilon \bar{f}^{h-1} h\left\|\mathcal{A}_{0}\right\|_{\mathcal{H}^{s}, \mathcal{H}^{s-q}} \prod_{l=1}^{h}\left\|\mathbf{u}_{l}\right\|_{\mathcal{H}^{s}} .
\end{aligned}
$$

Since $\epsilon>0$ can be taken arbitrarily small, and $\mathcal{A}_{0}\left(\mathbf{u}_{1}^{\prime}, \ldots, \mathbf{u}_{h}^{\prime}\right)$, given by (5.13), coincides with the sum in (5.1), we obtain that the limit in (5.4) as $\bar{d} \rightarrow 0$ exists. Since also $f_{l}(\mathbf{M}) \mathbf{w}_{l} \rightarrow f_{l}(\mathbf{M}) \mathbf{u}_{l}$ as $\omega_{l}^{-}, \omega_{l}^{+} \rightarrow \infty$, and $\mathcal{A}_{0}$ is continuous, we obtain (5.7).

In what follows, we give another sufficient condition for the existence of $\mathcal{A}_{M, \chi}\left(\mathbf{u}_{1}, \ldots, \mathbf{u}_{h}\right)$. Denote

$$
\vec{z}=\left(z_{1}, \ldots, z_{h}\right), \quad d z_{1} \cdots d z_{h}=d^{h} \vec{z}, \quad \vec{\omega}=\left(\omega_{1}, \ldots, \omega_{h}\right) .
$$

Let $\mathbf{K}\left(z_{1}, \ldots, z_{h}\right)=\mathbf{K}(\vec{z})$ be a Bochner-integrable function on $\mathbb{R}^{h}$ with values in the Banach space of bounded $h$-linear operators from $\left(\mathcal{H}^{s}\right)^{h}$ into $\mathcal{H}^{s-q}$ with operator norm $\|\cdot\|_{\mathcal{H}^{s}, \mathcal{H}^{s-q}}$. Recall that a function $\mathbf{X}(\vec{z})$ with values in a Banach space $\mathcal{B}$ is Bochner integrable if there exists a sequence of countably-valued functions $\left\{\mathbf{X}_{n}(\vec{z})\right\}$ converging almost everywhere to $\mathbf{X}(\vec{z})$ and satisfying

$$
\int_{\mathbb{R}^{h}}\left\|\mathbf{X}_{n}(\vec{z})-\mathbf{X}(\vec{z})\right\|_{\mathcal{B}} d^{h} \vec{z} \rightarrow 0
$$

(see, for example, [17] for properties of the Bochner integral). Assume that

$$
\int_{\mathbb{R}^{h}}\left\|\mathbf{K}\left(z_{1}, \ldots, z_{h}\right)\right\|_{\mathcal{H}^{s}, \mathcal{H}^{s-q}} d z_{1} \cdots d z_{h} \leq C_{0 s \mathbf{K}}
$$

Now let the $h$-linear operator $\chi_{\mathbf{K}}\left(\omega_{1}, \ldots, \omega_{h}, \mathbf{u}_{1}, \ldots, \mathbf{u}_{h}\right)$ be the Fourier transform of $\mathbf{K}(\vec{z} ; \overrightarrow{\mathbf{u}})$ :

$$
\chi_{\mathbf{K}}(\vec{\omega}, \overrightarrow{\mathbf{u}})=\int_{\mathbb{R}^{h}} e^{\left(i \sum_{l=1}^{h} \omega_{l} z_{l}\right)} \mathbf{K}(\vec{z} ; \overrightarrow{\mathbf{u}}) d^{h} \vec{z} .
$$

where the integral is understood in the Bochner sense.

Theorem 5.3. Let $\mathbf{K}(\vec{z})$ be Bochner integrable and let (5.16) hold. Then $\chi_{\mathbf{K}}(\vec{\omega}, \overrightarrow{\mathbf{u}})$ defined by (5.17) is a continuous function of $\vec{\omega}$ with values in the space of multilinear operators from $\mathcal{H}^{s}$ to $\mathcal{H}^{s-q}$. In addition to that, the limit (5.4), where $\chi=\chi_{\mathbf{K}}$ is defined by (5.17), exists in the norm of $\mathcal{H}^{s-q}$ and it determines a bounded $h$-linear operator $\mathcal{A}_{\mathbf{M}, \chi_{K}}$ given by (5.5) with $\chi=\chi_{\mathbf{K}}$. This operator can be written in the form

$$
\mathcal{A}_{\mathbf{M}, \chi_{K}}\left(\mathbf{u}_{1}, \ldots, \mathbf{u}_{h}\right)=\int_{\mathbb{R}^{h}} \mathbf{K}\left(\vec{z} ; e^{i z_{1} \mathbf{M}} \mathbf{u}_{1}, \ldots, e^{i z_{h} \mathbf{M}} \mathbf{u}_{h}\right) d^{h} \vec{z}
$$


for every $\mathbf{u}_{1}, \ldots, \mathbf{u}_{h} \in \mathcal{H}^{s}$. The right-hand side of (5.18) is defined as a Bochner integral of the $\mathcal{H}^{s-q}$-valued function of $\vec{z}$.

Proof. Let us fix $\mathbf{u}_{1}, \ldots, \mathbf{u}_{h} \in \mathcal{H}^{s}$ with $\left\|\mathbf{u}_{l}\right\|_{\mathcal{H}^{s}}^{2} \leq 1, l=1, \ldots, h$. The continuity of $\chi_{\mathbf{K}}(\vec{\omega}, \overrightarrow{\mathbf{u}})$ with respect to $\vec{\omega}$ can be proven exactly as the continuity of the Fourier transform of scalar-valued functions from $L_{1}\left(\mathbb{R}^{h}\right)$. Now we would like to check that the right-hand side of (5.18) is well defined. First, we consider $\left|\vec{z}_{l}\right| \leq \bar{Z}$, that is, $\left|z_{l}\right| \leq \bar{Z}, l=1, \ldots, h$. Note that the integral

$$
\int_{\left|z_{l}\right| \leq \bar{Z}} \mathbf{K}\left(\vec{z} ; e^{i z_{1} \mathbf{M}} \mathbf{u}_{1}, \ldots, e^{\left(i z_{h} \mathbf{M}\right)} \mathbf{u}_{h}\right) d^{h} \vec{z}
$$

is well defined in Bochner sense. Indeed, $e^{i z_{l} \mathbf{M}}$ is a unitary operator; therefore

$$
\begin{aligned}
& \int_{|\vec{z}| \leq \bar{Z}}\left\|\mathbf{K}\left(\vec{z} ; e^{i z_{1} \mathbf{M}} \mathbf{u}_{1}, \ldots, e^{i z_{h} \mathbf{M}} \mathbf{u}_{h}\right)\right\|_{\mathcal{H}^{s-q}} d^{h} \vec{z} \\
& \quad \leq \int_{|\vec{z}| \leq \bar{Z}}\|\mathbf{K}(\vec{z} ; \cdot)\|_{\mathcal{H}^{s}, \mathcal{H}^{s-q}} d^{h} \vec{z} .
\end{aligned}
$$

To check Bochner integrability we have only to show (see $[\mathbf{1 7}]$ ) that

$$
\mathbf{K}\left(\vec{z} ; e^{i z_{1} \mathbf{M}} \mathbf{u}_{1}, \ldots, e^{i z_{h} \mathbf{M}} \mathbf{u}_{h}\right)
$$

can be $L_{1}$-approximated by measurable functions of $\vec{z}$ that take a countable set of values. Since the operator $\mathbf{K}(\vec{z} ; \cdot)$ is Bochner integrable by assumption, it can be approximated by operator-valued functions $\mathbf{k}(\vec{z} ; \cdot)$ such that

$$
\int\|\mathbf{K}(\vec{z} ; \cdot)-\mathbf{k}(\vec{z} ; \cdot)\|_{\mathcal{H}^{s}, \mathcal{H}^{s-q}} d z \leq \epsilon_{1}
$$

with arbitrary small $\epsilon_{1}$. Therefore, it suffices to approximate $e^{\left(i z_{l} \mathbf{M}\right)} \mathbf{u}_{l}$ by a function $\mathbf{v}_{l}\left(z_{l}\right)$ that takes a countable set of values and such that $\left\|e^{i z_{l} \mathbf{M}_{\mathbf{u}}} \mathbf{u}_{l}-\mathbf{v}_{l}\left(z_{l}\right)\right\| \leq \epsilon_{1}$. This can be easily done in two steps. First, we use (5.9) to approximate $\mathbf{u}_{l}$ by $\mathbf{w}_{l}=$ $\left[\mathcal{E}\left(\omega^{+}\right)-\mathcal{E}\left(-\omega^{-}\right)\right] \mathbf{u}_{l}$. Second, we use uniform continuity of $e^{\left(i z_{l} \mathbf{M}\right)} \mathbf{w}_{l}$ with respect to $z_{l}$ to approximate $e^{\left(i z_{l} \mathbf{M}\right)} \mathbf{w}_{l}$ by $\mathbf{v}_{l}\left(z_{l}\right)$. Therefore (5.19) exists, and sending $\bar{Z}$ to infinity, we see from (5.16) that the right-hand side of (5.18) exists in Bochner sense too.

The integral sum (5.1) for $\mathcal{A}_{\mathbf{M}, \chi_{K}}$ defined by (5.4), where $\chi=\chi_{\mathbf{K}}$ is defined by $(5.17)$ and $-\omega_{l}^{-} \leq \omega_{l, i_{l}} \leq \omega_{l}^{+}$, takes the form

$$
\begin{aligned}
\widetilde{\mathbf{I}} & {\left[\vec{z}, \overrightarrow{\mathbf{u}}, \vec{\omega}^{\prime}, \vec{\omega}^{+}, \vec{\omega}^{-}\right] } \\
& =\sum_{i_{1}, \ldots, i_{h}} \int_{\mathbb{R}^{h}} e^{i \omega_{1, i_{1}}^{\prime} z_{1}} \cdots e^{i \omega_{h, i_{h}}^{\prime} z_{h}} \mathbf{K}\left(\vec{z} ; \Delta_{i_{1}} \mathcal{E}(\omega) \mathbf{u}_{1}, \ldots, \Delta_{i_{h}} \mathcal{E}(\omega) \mathbf{u}_{h}\right) d^{h} \vec{z} \\
& =\sum_{i_{1}, \ldots, i_{h}} \int_{\mathbb{R}^{h}} \mathbf{K}\left(\vec{z} ; \Delta_{i_{1}} \mathcal{E}(\omega) e^{i \omega_{1, i_{1}}^{\prime} z_{1}} \mathbf{u}_{1}, \ldots, \Delta_{i_{h}} \mathcal{E}(\omega) e^{i \omega_{h, i_{h}}^{\prime} z_{h}} \mathbf{u}_{h}\right) d^{h} \vec{z} \\
& =\int_{\mathbb{R}^{h}} \mathbf{K}\left(\vec{z} ; \sum_{i_{1}} \Delta_{i_{1}} \mathcal{E}(\omega) e^{i \omega_{1, i_{1}}^{\prime} z_{1}} \mathbf{u}_{1}, \ldots, \sum_{i_{h}} \Delta_{i_{h}} \mathcal{E}(\omega) e^{i \omega_{h, i_{h}}^{\prime} z_{h}} \mathbf{u}_{h}\right) d^{h} \vec{z} .
\end{aligned}
$$


We denote the integrand of the last integral by

$$
\begin{aligned}
\widetilde{\mathbf{K}}[\vec{z} & \left., \overrightarrow{\mathbf{u}}, \vec{\omega}^{\prime}, \vec{\omega}^{+}, \vec{\omega}^{-}\right] \\
& =\mathbf{K}\left(\vec{z} ; \sum_{i_{1}} \Delta_{i_{1}} \mathcal{E}(\omega) e^{i \omega_{1, i_{1}}^{\prime} z_{1}} \mathbf{u}_{1}, \ldots, \sum_{i_{h}} \Delta_{i_{h}} \mathcal{E}(\omega) e^{i \omega_{h, i_{h}}^{\prime} z_{h}} \mathbf{u}_{h}\right)
\end{aligned}
$$

therefore

$$
\widetilde{\mathbf{I}}\left[\vec{z}, \overrightarrow{\mathbf{u}}, \vec{\omega}^{\prime}, \vec{\omega}^{+}, \vec{\omega}^{-}\right]=\int_{\mathbb{R}^{h}} \widetilde{\mathbf{K}}\left[\vec{z}, \overrightarrow{\mathbf{u}}, \vec{\omega}^{\prime}, \vec{\omega}^{+}, \vec{\omega}^{-}\right] d^{h} \vec{z}
$$

Let

$$
\begin{aligned}
\mathbf{K} & {\left[\vec{z}, \overrightarrow{\mathbf{u}}, \vec{\omega}^{+}, \vec{\omega}^{-}\right] } \\
& =\mathbf{K}\left(\vec{z}, e^{i z_{1} \mathbf{M}}\left[\mathcal{E}\left(\omega_{1}^{+}\right)-\mathcal{E}\left(-\omega_{1}^{-}\right)\right] \mathbf{u}_{1}, \ldots, e^{i z_{h} \mathbf{M}}\left[\mathcal{E}\left(\omega_{h}^{+}\right)-\mathcal{E}\left(-\omega_{h}^{-}\right)\right] \mathbf{u}_{h}\right)
\end{aligned}
$$

and

$$
\mathbf{I}\left[\vec{z}, \overrightarrow{\mathbf{u}}, \vec{\omega}^{+}, \vec{\omega}^{-}\right]=\int_{\mathbb{R}^{h}} \mathbf{K}\left[\vec{z}, \overrightarrow{\mathbf{u}}, \vec{\omega}^{+}, \vec{\omega}^{-}\right] d^{h} \vec{z}
$$

Note that

$$
\begin{aligned}
\left\|\sum_{i_{l}} \Delta_{i_{l}} \mathcal{E}(\omega) e^{i \omega_{l}^{\prime} z_{l}} \mathbf{u}_{l}\right\|_{\mathcal{H}^{s}}^{2} & =\sum_{i_{l}}\left\|\Delta_{i_{l}} \mathcal{E}(\omega) e^{i \omega_{l}^{\prime} z_{l}} \mathbf{u}_{l}\right\|_{\mathcal{H}^{s}}^{2} \\
& =\sum_{i_{l}}\left\|\Delta_{i_{l}} \mathcal{E}(\omega) \mathbf{u}_{l}\right\|^{2} \leq\left\|\mathbf{u}_{l}\right\|_{\mathcal{H}^{s}}^{2}
\end{aligned}
$$

and similarly for $e^{i z_{1} \mathbf{M}}\left[\mathcal{E}\left(\omega_{l}^{+}\right)-\mathcal{E}\left(-\omega_{l}^{-}\right)\right] \mathbf{u}_{l}$. Using (4.9), we obtain that

$$
\widetilde{\mathbf{I}}\left[\vec{z}, \overrightarrow{\mathbf{u}}, \vec{\omega}^{\prime}, \vec{\omega}^{+}, \vec{\omega}^{-}\right]-\mathbf{I}\left[\vec{z}, \overrightarrow{\mathbf{u}}, \vec{\omega}^{+}, \vec{\omega}^{-}\right]
$$

equals the sum of $h$ expressions of the form

$$
\int_{\mathbb{R}^{h}} \mathbf{K}\left(\vec{z} ; \mathbf{f}_{1}, \ldots, \mathbf{x}_{l}, \ldots, \mathbf{f}_{h}\right) d^{h} \vec{z}
$$

where the $l$ th argument $\mathbf{x}_{l}$ equals the difference of the corresponding arguments of $\widetilde{\mathbf{K}}\left[\vec{z}, \overrightarrow{\mathbf{u}}, \vec{\omega}^{\prime}, \vec{\omega}^{+}, \vec{\omega}^{-}\right]$and $\mathbf{K}\left[\vec{z}, \overrightarrow{\mathbf{u}}, \vec{\omega}^{+}, \vec{\omega}^{-}\right]$

$$
\mathbf{x}_{l}=\sum_{i_{l}} \Delta_{i_{l}} \mathcal{E}(\omega) e^{i \omega_{l, i_{l}}^{\prime} z_{1}} \mathbf{u}_{l}-e^{\left(i z_{1} \mathbf{M}\right)}\left[\mathcal{E}\left(\omega_{l}^{+}\right)-\mathcal{E}\left(-\omega_{l}^{-}\right)\right] \mathbf{u}_{l},
$$

and the remaining $\mathbf{f}_{i}, i \neq l$, coincide with their arguments. We have $\left\|\mathbf{f}_{i}\right\|_{\mathcal{H}^{s}}^{2} \leq 1, i \neq$ $l$ according to (5.23). Hence,

$$
\left\|\int_{\mathbb{R}^{h}} \mathbf{K}\left(\vec{z} ; \mathbf{f}_{1}, \ldots, \mathbf{x}_{l}, \ldots, \mathbf{f}_{h}\right) d^{h} \vec{z}\right\|_{\mathcal{H}^{s-q}} \leq \int_{\mathbb{R}^{h}}\|\mathbf{K}(\vec{z} ; \cdot)\|_{\mathcal{H}^{s}, \mathcal{H}^{s-q}}\left\|\mathbf{x}_{l}\right\|_{\mathcal{H}^{s}} d^{h} \vec{z}
$$

and

$$
\begin{aligned}
& \left\|\tilde{\mathbf{I}}\left[\vec{z}, \overrightarrow{\mathbf{u}}, \vec{\omega}^{\prime}, \vec{\omega}^{+}, \vec{\omega}^{-}\right]-\mathbf{I}\left[\vec{z}, \overrightarrow{\mathbf{u}}, \vec{\omega}^{+}, \vec{\omega}^{-}\right]\right\|_{\mathcal{H}^{s-q}} \\
& \quad \leq \int_{\mathbb{R}^{h}}\|\mathbf{K}(\vec{z} ; \cdot)\|_{\mathcal{H}^{s}, \mathcal{H}^{s-q}} \sum_{l}\left\|\mathbf{x}_{l}\right\|_{\mathcal{H}^{s}} d^{h} \vec{z} .
\end{aligned}
$$


Note that, for $|\vec{z}| \leq \bar{Z}$, we have

$$
\begin{aligned}
\left\|\mathbf{x}_{l}\right\|_{\mathcal{H}^{s}} & \leq\left\|\sum_{i_{l}} \Delta_{i_{l}} \mathcal{E}(\omega) e^{i \omega_{l}^{\prime} z_{l}} \mathbf{u}_{l}-e^{\left(i z_{l} \mathbf{M}\right)}\left[\mathcal{E}\left(\omega_{l}^{+}\right)-\mathcal{E}\left(-\omega_{l}^{-}\right)\right] \mathbf{u}_{l}\right\|_{\mathcal{H}^{s}} \\
& \leq \bar{Z} \bar{d}
\end{aligned}
$$

According to (5.16)

$$
\int_{|\vec{z}| \geq \bar{Z}}\|\mathbf{K}(\vec{z} ; \cdot)\|_{\mathcal{H}^{s}, \mathcal{H}^{s-q}} d^{h} \vec{z}=p(\bar{Z}) \rightarrow 0 \text { as } \bar{Z} \rightarrow \infty .
$$

Setting $\bar{Z}=1 / \sqrt{\bar{d}}$ we obtain

$$
\begin{aligned}
\int_{\mathbb{R}^{h}}\|\mathbf{K}(\vec{z} ; \cdot)\|_{\mathcal{H}^{s}, \mathcal{H}^{s-q}}\left\|\mathbf{x}_{l}\right\|_{\mathcal{H}^{s}} d^{h} \vec{z} \\
\leq \bar{d} \bar{Z} \int_{|\vec{z}| \leq \bar{Z}}\|\mathbf{K}(\vec{z} ; \cdot)\|_{\mathcal{H}^{s}, \mathcal{H}^{s-q}} d^{h} \vec{z} \\
\quad+\int_{|\bar{z}| \geq \bar{Z}}\|\mathbf{K}(\vec{z} ; \cdot)\|_{\mathcal{H}^{s}, \mathcal{H}^{s-q}}\left\|\mathbf{x}_{l}\right\|_{\mathcal{H}^{s}} d^{h} \vec{z} \\
\leq \bar{d} \bar{Z} C_{0 s \mathbf{K}}+2 p(\bar{Z}) C_{0 s \mathbf{K}}=C_{0 s \mathbf{K}}[\sqrt{\bar{d}}+2 p(1 / \sqrt{\bar{d}})] .
\end{aligned}
$$

From (5) we infer

$$
\begin{aligned}
& \left\|\widetilde{\mathbf{I}}\left[\vec{z}, \overrightarrow{\mathbf{u}}, \vec{\omega}^{\prime}, \vec{\omega}^{+}, \vec{\omega}^{-}\right]-\mathbf{I}\left[\vec{z}, \overrightarrow{\mathbf{u}}, \vec{\omega}^{+}, \vec{\omega}^{-}\right]\right\|_{\mathcal{H}^{s-q}} \\
& \quad \leq h C_{0 s \mathbf{K}}[\sqrt{\bar{d}}+2 p(1 / \sqrt{\bar{d}})] .
\end{aligned}
$$

Since $\sqrt{\bar{d}}+2 p(1 / \sqrt{\bar{d}}) \rightarrow 0$ as $\bar{d} \rightarrow 0$, we conclude that the limit as $\bar{d} \rightarrow 0$ of the integral sums with fixed $\omega_{l}^{+}, \omega_{l}^{-}$equals

$$
\int_{|\vec{z}| \leq \bar{Z}} \mathbf{K}\left(\vec{z} ; e^{i z_{1} \mathbf{M}}\left[\mathcal{E}\left(\omega_{1}^{+}\right)-\mathcal{E}\left(-\omega_{1}^{-}\right)\right] \mathbf{u}_{1}, \ldots, e^{i z_{h} \mathbf{M}}\left[\mathcal{E}\left(\omega_{h}^{+}\right)-\mathcal{E}\left(-\omega_{h}^{-}\right)\right] \mathbf{u}_{h}\right) d^{h} \vec{z}
$$

After that we let $\omega_{l}^{+} \rightarrow \infty, \omega_{l}^{-} \rightarrow \infty$ and get

$$
\left\|e^{\left(i z_{l} \mathbf{M}\right)} \mathbf{u}_{l}-e^{\left(i z_{l} \mathbf{M}\right)}\left[\mathcal{E}\left(\omega_{l}^{+}\right)-\mathcal{E}\left(-\omega_{l}^{-}\right)\right] \mathbf{u}_{l}\right\|_{\mathcal{H}^{s}} \rightarrow 0, l=1, \ldots, h .
$$

According to (5.16), the integral

$$
\int_{\mathbb{R}^{h}} \mathbf{K}\left[\vec{z}, \overrightarrow{\mathbf{u}}, \vec{\omega}^{+}, \vec{\omega}^{-}\right] d^{h} \vec{z}
$$

in $\mathcal{H}^{s-q}$ depends continuously on $\left[\mathcal{E}\left(\omega_{l}^{+}\right)-\mathcal{E}\left(-\omega_{l}^{-}\right)\right] \mathbf{u}_{l} \in \mathcal{H}^{s}$, and hence, using (5.25), we obtain (5.18) in the limit.

REMARK 5.4. Obviously, Theorem 5.3 is applicable to operators $\chi(\vec{\omega}, \overrightarrow{\mathbf{u}})$ of the form $f_{1}\left(\omega_{1}\right) \cdots f_{h}\left(\omega_{h}\right) \mathcal{A}_{0}(\overrightarrow{\mathbf{u}})$ considered in Theorem 5.2. One must take into account that conditions on $f_{l}(\omega)$ in Theorem 5.2 are less restrictive, since not every continuous bounded function $f$ is a Fourier transform of an integrable function in $L_{1}(\mathbb{R})$. 
Now we would like to apply Theorem 5.3 to $\overline{\mathcal{K}}(\overrightarrow{\mathbf{u}})$ given by (4.16). First we formulate the definition extending the standard definition of susceptibility in the frequency domain directly to the classes introduced of operators satisfying (4.3).

Definition 5.5. The susceptibility symbol $\chi_{\mathbf{K}_{0}}(\vec{\omega})=\chi_{\mathbf{K}_{0}}(\vec{\omega} ; \cdot)$ depending on the frequencies $\vec{\omega}=\left(\omega_{1}, \ldots, \omega_{h}\right)$ is the Fourier transform of the operator $\mathbf{K}_{0}^{(h)}\left(t_{1}, \ldots, t_{h} ; \cdot\right)\left(\mathbf{K}_{0}^{(h)}\left(t_{1}, \ldots, t_{h}\right)\right.$ coincides with $\mathbf{K}^{(h)}\left(t_{1}, \ldots, t_{h} ; \cdot\right)$ for $t_{1}, \ldots, t_{h} \geq$ 0 , and is extended by zero if one of the $t_{l}$ is $\left.<0\right)$ :

$$
\chi_{\mathbf{K}_{0}}(\vec{\omega})=\int_{0}^{\infty} \cdots \int_{0}^{\infty} \mathbf{K}^{(h)}\left(t_{1}, \ldots, t_{h}\right) e^{\left[i \sum_{l=1}^{h} \omega_{l} t_{l}\right]} d t_{1} \cdots d t_{h}
$$

Obviously, (2.20) is a particular case of (5.26).

Lemma 5.6. Let (4.1), (4.3) hold. Then

$$
\overline{\mathcal{K}}(\overrightarrow{\mathbf{u}}, t)=\mathcal{K}_{\chi_{\mathbf{K}_{0}}}(\overrightarrow{\mathbf{v}}, t),
$$

where $\overline{\mathcal{K}}(\overrightarrow{\mathbf{u}}, t)$ is defined by (4.16),

$$
\begin{aligned}
& \mathcal{K}_{\chi_{\mathbf{K}_{0}}}(\overrightarrow{\mathbf{v}}, t) \\
& \quad=\int_{-\infty}^{\infty} \ldots \int_{-\infty}^{\infty} e^{\left[-i \sum_{l=1}^{h} \omega_{l} t\right]} \chi_{\mathbf{K}_{0}}\left(\vec{\omega} ; d \mathcal{E}\left(\omega_{1}\right) \mathbf{v}_{1}(\varrho t), \ldots, d \mathcal{E}\left(\omega_{h}\right) \mathbf{v}_{h}(\varrho t)\right) .
\end{aligned}
$$

Proof. First of all we note that the Fourier transform of $\mathbf{K}_{0 t}^{(h)}\left(t_{1}, \ldots, t_{h}\right)=$ $\mathbf{K}_{0}^{(h)}\left(t_{1}-t, \ldots, t_{h}-t\right)$ equals the Fourier transform of $\mathbf{K}_{0}^{(h)}\left(t_{1}, \ldots, t_{h}\right)$ times $e^{\left[-i \sum_{l=1}^{h} \omega_{l} t\right]}$. We set $\mathbf{u}_{l}=\mathbf{u}_{l}(t, \tau)$ and rewrite (4.16) in the form

$$
\mathcal{K}_{\chi}(\overrightarrow{\mathbf{u}}(t, \tau), t)=\int_{\mathbb{R}^{h}} \mathbf{K}^{(h)}\left(z_{1}, \ldots, z_{h} ; e^{\left[i \mathbf{M} z_{1}\right]} \mathbf{u}_{1}, \ldots, e^{\left[i \mathbf{M} z_{h}\right]} \mathbf{u}_{h}\right) d z_{1} \cdots d z_{h}
$$

This expression has the form of the right-hand side of (5.18). By Theorem 5.3 we obtain

$$
\overline{\mathcal{K}}(\overrightarrow{\mathbf{u}}, t)=\int_{-\infty}^{\infty} \cdots \int_{-\infty}^{\infty} \chi_{\mathbf{K}_{0 t}}\left(\vec{\omega}, d \mathcal{E}\left(\omega_{1}\right) \mathbf{u}_{1}, \ldots, d \mathcal{E}\left(\omega_{h}\right) \mathbf{u}_{h}\right)
$$

Using the definition of $\mathbf{u}_{l}(t, \tau)$ in (4.15) we obtain (5.27).

From Lemmas 4.1 and 5.6 we immediately get the following theorem on the representation of $\overline{\mathcal{K}}(\overrightarrow{\mathbf{u}}, t)$ in terms of the susceptibility $\chi_{\mathbf{K}_{0}}$.

Theorem 5.7. Let (4.3), (4.7) and (4.8) hold. Let $\mathcal{K}(\overrightarrow{\mathbf{u}}, t)$ be defined by (4.2), (4.1) with $\mathbf{j}_{l}(\tau)=\mathbf{j}^{0}(\tau)$ given by (3.17), where $\mathbf{j}\left(\tau^{\prime}\right)$ satisfies (3.12). Then

$$
\mathcal{K}(\overrightarrow{\mathbf{u}}, t)=\mathcal{K}_{\chi}(\overrightarrow{\mathbf{u}}, t)+\mathbf{O}(\varrho),
$$

where $\chi=\chi_{\mathbf{K}_{0}}, \mathcal{K}_{\chi}(\overrightarrow{\mathbf{u}}, t)$ is given by (5.6), and

$$
\|\mathbf{O}(\varrho)\|_{\mathcal{H}^{s-q}} \leq C \varrho .
$$

Proof. By (3.17) and (3.12), Lipschitz condition (4.6) holds and we can apply Lemma 4.1. We use Lemma 5.6 to write down $\overline{\mathcal{K}}(\overrightarrow{\mathbf{u}}, t)$ and obtain (5.28). 


\section{Maxwell operator with periodic coefficients}

In this section, for completeness, we consider the case where $\mathbf{M}$ has periodic coefficients. In this case the spectral theory of the Maxwell operator $\mathbf{M}$ turns into the Floquet-Bloch theory, which is much more specific than the general one. It allows us not only to separate the time dependence, but also the large-scale spatial dependence.

We describe the basic spectral properties of the operator $\mathbf{M}$ for the dimension $d=3$. For $d=2$ or $d=1$ one must consider vector fields which do not depend on $x_{3}$ if $d=2$ or $x_{2}, x_{3}$ if $d=1$.

In the case of a $\mathbb{Z}^{3}$-periodic $\eta^{(1)}(\mathbf{r})$, the Floquet-Bloch theory (see [2], [15], $[\mathbf{2 1}],[\mathbf{2 5}],[\mathbf{3 1}])$ describes the spectrum and the eigenmodes of the operator $\mathbf{M}$ as follows. We introduce the operator

$$
\widetilde{\mathfrak{m}}(\mathbf{k}) \tilde{\Psi}(\mathbf{r})=\nabla \times \mu^{-1} \eta^{(1)}(\mathbf{r}) \nabla \times \tilde{\Psi}(\mathbf{r}), \nabla \cdot \tilde{\Psi}(\mathbf{r})=0, \mathbf{r} \text { in }[0,1]^{3},
$$

where $\tilde{\Psi}(\mathbf{r})$ satisfies the following cyclic Bloch boundary conditions:

$$
\begin{aligned}
\tilde{\Psi}(\mathbf{r}+\mathbf{m}) & =\exp (i \mathbf{m} \cdot \mathbf{k}) \tilde{\Psi}(\mathbf{r}), \\
\nabla \tilde{\Psi}(\mathbf{r}+\mathbf{m}) & =\exp (i \mathbf{m} \cdot \mathbf{k}) \nabla \tilde{\Psi}(\mathbf{r}), \mathbf{m} \text { in } \mathbf{Z}^{3} .
\end{aligned}
$$

The eigenmodes $\tilde{\mathbf{g}}_{n}(\mathbf{r}, \mathbf{k})$ of the operator $\widetilde{\mathfrak{m}}(\mathbf{k})$ have the Bloch form

$$
\tilde{\mathbf{g}}_{n}(\mathbf{r}, \mathbf{k})=e^{\{i \mathbf{k} \cdot \mathbf{r}\}} \hat{\mathbf{g}}_{n}(\mathbf{r}, \mathbf{k}) \text {, where } \hat{\mathbf{g}} \text { is a } \mathbb{Z}^{3} \text {-periodic function of } \mathbf{r} \text {. }
$$

The set of all the eigenvalues and eigenmodes of the operator $\widetilde{\mathfrak{m}}$ (direct integral of $\widetilde{\mathfrak{m}}(\mathbf{k}))$ can be parametrized by two indices: $n=1,2, \ldots$, called the zone number (or band number), and the continuous $\mathbf{k}=\left(k_{1}, \ldots, k_{d}\right)$, called the quasimomentum, from the base Brillouin zone $[-\pi, \pi]^{3}$. The eigenvalue and the eigenmode of $\tilde{\mathfrak{m}}$ associated with given $n$ and $\mathbf{k}$ are denoted by $\omega_{n}^{2}(\mathbf{k})$ (it is the square of the angular frequency) and $\tilde{\mathbf{g}}_{n}(\mathbf{r}, \mathbf{k})$, respectively, of the Bloch form (6.4). They satisfy the equation

$$
\widetilde{\mathfrak{m}}(\mathbf{k}) \tilde{\mathbf{g}}_{n}(\mathbf{r}, \mathbf{k})=\omega_{n}^{2}(\mathbf{k}) \tilde{\mathbf{g}}_{n}(\mathbf{r}, \mathbf{k}), \nabla \cdot \tilde{\mathbf{g}}_{n}(\mathbf{r}, \mathbf{k})=0, \mathbf{r} \text { in }[0,1]^{3},
$$

where

$$
0 \leq \omega_{1}^{2}(\mathbf{k}) \leq \omega_{2}^{2}(\mathbf{k}) \leq \cdots
$$

and the following Bloch boundary conditions hold:

$$
\begin{aligned}
\tilde{\mathbf{g}}_{n}(\mathbf{r}+\mathbf{m}, \mathbf{k}) & =\exp \{i \mathbf{k} \cdot \mathbf{m}\} \tilde{\mathbf{g}}_{n}(\mathbf{r}, \mathbf{k}), \\
\nabla \tilde{\mathbf{g}}_{n}(\mathbf{r}+\mathbf{m}, \mathbf{k}) & =\exp \{i \mathbf{k} \cdot \mathbf{m}\} \nabla \tilde{\mathbf{g}}_{n}(\mathbf{r}, \mathbf{k}), \mathbf{m} \text { in } \mathbb{Z}^{3} .
\end{aligned}
$$

The eigenvalues of the Maxwell operator $\mathbf{M}$ are $\pm \omega_{n}(\mathbf{k})$, with respective eigenfunctions $\tilde{\mathbf{G}}_{ \pm 1, n}(\mathbf{r}, \mathbf{k})$. Since the index of the eigenmodes $\tilde{\mathbf{G}}_{ \pm 1, n}(\mathbf{r}, \mathbf{k})$ is always a pair $\bar{n}=(\zeta, n)$ with $\zeta= \pm 1$, we abbreviate the notation by introducing

$$
\bar{n}=(\zeta, n), \text { where } \zeta= \pm 1, n=1,2, \ldots
$$

We set

$$
\omega_{\bar{n}}(\mathbf{k})=\zeta \omega_{n}(\mathbf{k}), \text { for } \bar{n}=(\zeta, n) .
$$

The vector fields $\tilde{\mathbf{G}}_{\bar{n}}(\mathbf{r}, \mathbf{k})$ have the following $\mathbf{D}$ and $\mathbf{B}$ components:

$$
\tilde{\mathbf{G}}_{\bar{n}}(\mathbf{r}, \mathbf{k})=\left[\begin{array}{c}
\tilde{\mathbf{D}}_{\bar{n}}(\mathbf{r}, \mathbf{k}) \\
\tilde{\mathbf{B}}_{\bar{n}}(\mathbf{r}, \mathbf{k})
\end{array}\right]=\left[\begin{array}{c}
i \zeta\left[\omega_{\bar{n}}(\mathbf{k}) \mu\right]^{-1} \nabla \times \tilde{\mathbf{g}}_{n}(\mathbf{r}, \mathbf{k}) \\
\tilde{\mathbf{g}}_{n}(\mathbf{r}, \mathbf{k})
\end{array}\right] .
$$


We have

$$
\begin{aligned}
\mathbf{M} \tilde{\mathbf{G}}_{\bar{n}}(\mathbf{r}, \mathbf{k}) & =\omega_{\bar{n}}(\mathbf{k}) \tilde{\mathbf{G}}_{\bar{n}}(\mathbf{r}, \mathbf{k}), \\
\tilde{\mathbf{G}}_{\bar{n}}(\mathbf{r}+\mathbf{m}, \mathbf{k}) & =\exp \{i \mathbf{k} \cdot \mathbf{m}\} \tilde{\mathbf{G}}_{\bar{n}}(\mathbf{r}, \mathbf{k}), \mathbf{m} \text { in } \mathbb{Z}^{3} .
\end{aligned}
$$

Clearly both components of $\tilde{\mathbf{G}}_{\bar{n}}(\mathbf{r}, \mathbf{k})$ are divergence-free.

The dependence of $\omega_{n}(\mathbf{k})$ on $\mathbf{k}$ is Lipschitzian. It must be noted that the issue of differentiability of eigenvalues is far from trivial, [20], Sections II.5, II.7, especially in the case of several parameters. All the difficulties are related to multiple eigenvalues. It turns out that in the analysis for the space dimensions $d=1,2,3$, in a generic situation it is sufficient to consider only the multiplicity 2 ; for a discussion see $[5]$.

We assume that the eigenvalues are naturally ordered,

$$
\omega_{1}(\mathbf{k}) \leq \omega_{2}(\mathbf{k}) \leq \cdots .
$$

If for a given $\mathbf{k}_{0}$ the eigenvalue $\omega_{n}\left(\mathbf{k}_{0}\right)$ is simple, i.e., of multiplicity one, then both $\omega_{n}(\mathbf{k})$ and $\mathbf{g}_{n}(\mathbf{r}, \mathbf{k})$ depend analytically on $\mathbf{k}$ in a neighborhood of the point $\mathbf{k}_{0}$. In the case where $\mathbf{k}_{0}$ is not a simple point the situation becomes more intricate and depends on the dimension of $\mathbf{k}$; for a discussion see [5].

The eigenfunctions $\tilde{\mathbf{G}}_{\bar{n}}(\mathbf{r}, \mathbf{k})$ with different $\bar{n}$ are orthogonal in the Hilbert space $\mathcal{H}$ defined by (2.5) and form an orthogonal basis in this space for every $\mathbf{k}$. Using standard properties of the Floquet-Bloch transform we get the following representation for an arbitrary vector field $\mathbf{U}=\left[\begin{array}{l}\mathbf{D} \\ \mathbf{B}\end{array}\right]$ with $\nabla \cdot \mathbf{D}=\mathbf{0}, \nabla \cdot \mathbf{B}=\mathbf{0}$ and finite energy $(\mathbf{U}, \mathbf{U})_{\mathcal{H}}$ :

$$
\mathbf{U}(\mathbf{r})=\frac{1}{(2 \pi)^{d}} \int_{[-\pi, \pi]^{3}} \tilde{\mathbf{U}}(\mathbf{r}, \mathbf{k}) d \mathbf{k}=\frac{1}{(2 \pi)^{d}} \sum_{\bar{n}} \int_{[-\pi, \pi]^{d}} \tilde{U}_{\bar{n}}(\mathbf{k}) \tilde{\mathbf{G}}_{\bar{n}}(\mathbf{r}, \mathbf{k}) d \mathbf{k}
$$

where

$$
\begin{aligned}
\tilde{\mathbf{U}}(\mathbf{r}, \mathbf{k}) & =\sum_{\bar{n}} \tilde{\mathbf{U}}_{\bar{n}}(\mathbf{r}, \mathbf{k}), \tilde{\mathbf{U}}_{\bar{n}}(\mathbf{r}, \mathbf{k})=\tilde{\Pi}_{\bar{n}}(\mathbf{k}) \tilde{\mathbf{U}}(\mathbf{r}, \mathbf{k})=\tilde{U}_{\bar{n}}(\mathbf{k}) \tilde{\mathbf{G}}_{\bar{n}}(\mathbf{r}, \mathbf{k}), \\
\tilde{U}_{\bar{n}}(\mathbf{k}) & =\left(\tilde{\mathbf{U}}(\cdot, \mathbf{k}), \tilde{\mathbf{G}}_{\bar{n}}(\cdot, \mathbf{k})\right)_{\mathcal{H}}\left(\left(\tilde{\mathbf{G}}_{\bar{n}}(\cdot, \mathbf{k}), \tilde{\mathbf{G}}_{\bar{n}}(\cdot, \mathbf{k})\right)_{\mathcal{H}}\right)^{-\frac{1}{2}}
\end{aligned}
$$

and $(\cdot, \cdot)_{\mathcal{H}}$ is defined by $(2.5) ; \tilde{\mathbf{G}}_{\bar{n}}$ are defined by $(6.10)$.

The exponent of $\mathbf{M}$ is given by

$$
e^{[i \mathbf{M} t]} \mathbf{U}_{0}(\mathbf{r})=\frac{1}{(2 \pi)^{d}} \int_{[-\pi, \pi]^{3}} \sum_{\bar{n}} e^{\left[i \omega_{\bar{n}}(\mathbf{k}) t\right]} \tilde{\mathbf{U}}(\mathbf{r}, \mathbf{k}) d \mathbf{k} .
$$

The wavepacket excitations $\mathbf{J}$ take the form $\mathbf{J}(\mathbf{r}, t, \tau)=e^{-i \mathbf{M} t} \mathbf{j}(\tau)$ and, as before, we assume that the currents vanish for negative times, i.e.,

$$
\mathbf{J}(\mathbf{r}, t)=0 \text { if } t \leq 0 .
$$

In addition, we assume that

$$
\|\mathbf{j}(\tau)\|_{\mathcal{H}^{s}} \leq \varrho C, \quad\|\mathbf{J}(t, \tau)\|_{\mathcal{H}^{s}} \leq \varrho C, \quad s>d / 2 .
$$

Having the Floquet-Bloch representation we get an analog of (3.13):

$$
\mathbf{J}(\mathbf{r}, t, \tau)=\frac{1}{(2 \pi)^{d}} \sum_{\bar{n}} \int \tilde{\mathbf{j}}_{n}(\mathbf{r}, \mathbf{k}, \tau) e^{-i \omega_{\bar{n}}(\mathbf{k}) t} d \mathbf{k}, \quad \tau=\varrho t
$$


where $\mathbf{j}(\mathbf{r}, \varrho t)$ gives a slow modulation of the time-harmonic carrier waves such that

$$
\begin{aligned}
\mathbf{j}(\mathbf{r}, \varrho t) & =\frac{1}{(2 \pi)^{d}} \sum_{\bar{n}} \int_{[-\pi, \pi]^{d}} \tilde{\mathbf{j}}_{n}(\mathbf{r}, \mathbf{k}, \tau) \tilde{\mathbf{G}}_{\bar{n}}(\mathbf{r}, \mathbf{k}) d \mathbf{k}, \\
\tilde{\mathbf{j}}_{n}(\mathbf{r}, \mathbf{k}, \tau) & =j_{n}(\mathbf{k}, \tau) \tilde{\mathbf{G}}_{\bar{n}}(\mathbf{r}, \mathbf{k}) .
\end{aligned}
$$

Then

$$
\tilde{\mathbf{U}}^{0}(\mathbf{r}, \mathbf{k}, t, \tau)=\sum_{\bar{n}} e^{\left\{-i \omega_{\bar{n}}(\mathbf{k}) t\right\}} \tilde{\mathbf{v}}_{\bar{n}}^{0}(\mathbf{r}, \mathbf{k}, t, \tau), \tau=\varrho t
$$

with

$$
\begin{aligned}
\tilde{\mathbf{v}}_{\bar{n}}^{0}(\mathbf{r}, \mathbf{k}, \tau) & =\int_{-\infty}^{\tau} \tilde{\mathbf{j}}_{n}\left(\mathbf{r}, \mathbf{k}, \tau^{\prime}\right) \mathbf{d} \tau^{\prime} \\
\mathbf{v}_{\bar{n}}^{0}(\mathbf{r}, t) & =\frac{1}{(2 \pi)^{d}} \int_{-\infty}^{t} \int \tilde{\mathbf{j}}_{n}\left(\mathbf{r}, \mathbf{k}, \tau^{\prime}\right) d \mathbf{k} \mathbf{d} \tau^{\prime} .
\end{aligned}
$$

Based on the Floquet-Bloch representation and using the expansion in the orthonormal eigenbasis $\mathbf{G}_{\bar{n}}$ in $\mathcal{H}$ we set similarly to (3.6)

$$
\begin{aligned}
\|\mathbf{V}\|_{\mathcal{H}^{s}}^{2} & =\sum_{\bar{n}} \int\left[\left|\omega_{\bar{n}}(\mathbf{k})\right|^{2 s}+1\right]\left|\tilde{V}_{\bar{n}}(\mathbf{k})\right|^{2} d \mathbf{k} \\
& =\sum_{\bar{n}} \iint_{[0,1]^{d}}\left[\left|\omega_{\bar{n}}(\mathbf{k})\right|^{2 s}+1\right]\left|\tilde{\mathbf{V}}_{\bar{n}}(\mathbf{r}, \mathbf{k}) ; \mathbf{r}, \sigma\right|^{2} d \mathbf{k d r} .
\end{aligned}
$$

For a periodic medium the susceptibilities defined in (2.20) are periodic:

$$
\chi^{(h)}(\mathbf{r}+\mathbf{m} ; \vec{\omega})=\chi^{(h)}(\mathbf{r} ; \omega), \mathbf{m} \text { in } \mathbb{Z}^{3}, h \geq 1 ; \quad \vec{\omega}=\left(\omega_{1}, \ldots, \omega_{h}\right) .
$$

The tensor $\vec{\chi}^{(h)}$ acts only on the second component of the vector $\mathbf{V}^{0}=\left[\begin{array}{l}\mathbf{V}_{D}^{0} \\ \mathbf{V}_{B}^{0}\end{array}\right]$, that is,

$$
\begin{aligned}
\vec{\chi}^{(h)} & \left(\mathbf{r} ; \omega_{\bar{n}^{\prime}}\left(\mathbf{k}^{\prime}\right), \ldots, \omega_{\bar{n}^{(h)}}\left(\mathbf{k}^{(h)}\right)\right) \prod_{j=1}^{h} \mathbf{V}_{\bar{n}^{(j)}}^{0}\left(\mathbf{k}^{(j)}, \varrho t\right) \\
= & \bar{\chi}^{(h)}\left(\mathbf{r} ; \omega_{\bar{n}^{\prime}}\left(\mathbf{k}^{\prime}\right), \ldots, \omega_{\bar{n}^{(h)}}\left(\mathbf{k}^{(h)}\right)\right) \prod_{j=1}^{h} \mathbf{V}_{D \bar{n}^{(j)}}^{0}\left(\mathbf{k}^{(j)}, \varrho t\right) .
\end{aligned}
$$

We also have the following representation of $\mathcal{S}_{\chi}^{(h)}$ in terms of the Floquet-Bloch transform:

$$
\begin{gathered}
\mathcal{S}_{\chi}^{(h)}(\mathbf{r}, t ; \mathbf{U}) \\
=\frac{1}{(2 \pi)^{h d}} \sum_{\bar{n}^{1}, \ldots, \bar{n}^{(h)}} \int \cdots \int e^{\left\{-i \sum \omega_{\bar{n}^{(j)}}\left(\mathbf{k}^{(j)}\right) t\right\}} \chi_{D}^{(h)}\left(\mathbf{r} ; \omega_{\bar{n}^{\prime}}\left(\mathbf{k}^{\prime}\right), \ldots, \omega_{\bar{n}^{(h)}}\left(\mathbf{k}^{(h)}\right)\right) \\
\times \prod_{j=1}^{h} \mathbf{V}_{D \bar{n}^{(j)}}\left(\mathbf{k}^{(j)}, \varrho t\right) d \mathbf{k}^{\prime} \cdots d \mathbf{k}^{(h-1)} .
\end{gathered}
$$

Note that the operator $\mathcal{S}_{\chi}^{(h)}$ has the form similar to (5.6). The difference is that the spectral projections $\mathcal{E}\left(\omega_{l}\right)$ are represented here in terms of the natural parametrization by the discrete parameter $\bar{n}^{(l)}$ and the continuous parameter $\mathbf{k}^{(l)}$. This special 
representation allows a detailed study of the nonlinear wave interactions as shown for the case $h=2$ in [5].

Applying the Floquet-Bloch transform we can recast (6.22) as

$$
\begin{gathered}
\widetilde{\mathcal{S}}_{\chi}^{(h)}(\mathbf{r}, \mathbf{k}, t ; \mathbf{U}) \\
=\sum_{\bar{n}^{1}, \ldots, \bar{n}^{(h)}} \int \cdots \int_{\mathbf{k}^{\prime}+\cdots+\mathbf{k}^{(h)}=\mathbf{k}} e^{\left\{-i \sum \omega_{\bar{n}^{(j)}}\left(\mathbf{k}^{(j)}\right) t\right\}} \chi_{D}^{(h)}\left(\mathbf{r} ; \omega_{\bar{n}^{\prime}}\left(\mathbf{k}^{\prime}\right), \ldots, \omega_{\bar{n}^{(h)}}\left(\mathbf{k}^{(h)}\right)\right) \\
\quad \times \prod_{j=1}^{h} \mathbf{V}_{D \bar{n}^{(j)}}\left(\mathbf{k}^{(j)}, \varrho t\right) \frac{1}{(2 \pi)^{(h-1) d}} d \mathbf{k}^{\prime} \cdots d \mathbf{k}^{(h-1)} .
\end{gathered}
$$

This representation shows that the phase matching condition $\mathbf{k}^{\prime}+\cdots+\mathbf{k}^{(h)}=\mathbf{k}$ holds explicitly for the wave interactions. It evidently stems from the medium periodicity and describes the behavior of waves on the spatial scale related to the period. For a detailed analysis of stronger nonlinear interactions selection rules see $[5]$.

Acknowledgment and Disclaimer. Effort of A. Babin and A. Figotin is sponsored by the Air Force Office of Scientific Research, Air Force Materials Command, USAF, under grant number F49620-01-1-0567. The US Government is authorized to reproduce and distribute reprints for governmental purposes notwithstanding any copyright notation thereon. The views and conclusions contained herein are those of the authors and should not be interpreted as necessarily representing the official policies or endorsements, either expressed or implied, of the Air Force Office of Scientific Research or the US Government.

\section{References}

[1] G. Agrawal, Nonlinear fiber optics, Academic Press, London, 1995.

[2] N. Ashcroft and N. Mermin, Solid state physics, Holt, Rinehart and Winston, New YorkLondon, 1976.

[3] G. Agrawal and R. Boyd, Contemporary nonlinear optics, Academic Press, 1992.

[4] W. Axmann and P. Kuchment, An efficient finite element method for computing spectra of photonic and acoustic band-gap materials, J. Comp. Phys. 150 (1999), pp. 468-481.

[5] A. Babin and A. Figotin, Nonlinear photonic crystals I. Quadratic nonlinearity, Waves in Random Media 11 (2001), R31-R102.

[6] — Nonlinear Maxwell equations in spatially inhomogeneous media, in preparation.

[7] C. G. Baldwin, An introduction to nonlinear optics, Plenum, New York, 1969.

[8] V. Belyakov, Diffraction optics of complex-structured periodic medium, Springer-Verlag, Berlin et al., 1992.

[9] M. Sh. Birman and M. Z. Solomyak, $L^{2}$-theory of the Maxwell operator in arbitrary domains, Russ. Math. Surveys 42 (1987), no. 6, 75-96.

[10] N. Bloembergen, Nonlinear optics, Benjamin, 1965.

[11] R. Boyd, Nonlinear optics, Academic Press, Londin, 1992.

[12] P. Butcher and D. Cotter, The elements of nonlinear optics, Cambridge Univ. Press, Cambridge, 1990.

[13] A. Douglis and L. Nirenberg, Interior estimates for elliptic systems of partial differential equations, Comm. Pure Appl. Math. 8 (1955), 503-538.

[14] L. Felsen and N. Marcuvits, Radiation and scattering of waves, Oxford University Press, IEEE Press, Oxford-New York, 1994.

[15] A. Figotin and A. Klein, Localization of classical waves II. Electromagnetic waves, Comm. Math. Phys. 184 (1997), 411-441.

[16] R. Harrington, Time-harmonic electromagnetic fields, McGraw-Hill, 1961.

[17] E. Hille and R. S. Phillips, Functional analysis and semigroups, Amer. Math. Soc., Providence, RI, 1957. 
[18] L. Hörmander, The analysis of linear partial differential operators. III. Pseudodifferential operators, Springer-Verlag, Berlin-New York, 1985.

[19] J. Jackson, Classical electrodynamics, Wiley, New York, 1975.

[20] T. Kato, Perturbation theory for linear operators, Springer-Verlag, Heidelberg-Berlin, 1995.

[21] P. Kuchment, Floquet theory for partial differential operators, Birkhäuser, Basel, 1993.

[22] D. Mills, Nonlinear optics, Springer-Verlag, Berlin et al., 1991.

[23] A. C. Newell and J. V. Moloney, Nonlinear optics, Addison-Wesley, Reading, MA, 1992.

[24] M. Reed and B. Simon, Methods of modern mathematical physics I. Functional analysis, Academic Press, New York-London, 1972.

[25] — Methods of modern mathematical physics. IV. Analysis of operators, Academic Press, New York-London, 1978.

[26] E. G. Sauter, Nonlinear optics, Wiley, New York, 1996.

[27] B.-W. Schulze, Boundary value problems and singular pseudo-differential operators, Wiley, New York, 1998.

[28] M. Shubin, Partial differential equations, VII. Spectral theory of differential operators, Encyclopaedia of Mathematical Sciences, Vol. 64, Springer-Verlag, Berlin, 1994.

[29] G. Whitham, Linear and nonlinear waves, Wiley, New York, 1974.

[30] J. T. Wloka, B. Rowley, and B. Lawruk, Boundary problems for elliptic systems, Cambridge Univ. Press, Cambridge, 1995.

[31] A. Yariv and P. Yeh, Optical waves in crystals, Wiley, New York, 1984.

Department of Mathematics, University of California at Irvine, Irvine, CA 926973875

E-mail address: ababine@math.uci.edu

Department of Mathematics, University of California at Irvine, Irvine, CA 926973875 .

E-mail address: afigotin@uci.edu 